\title{
EXACT BOUNDS FOR LINEAR OUTPUTS OF THE ADVECTION-DIFFUSION-REACTION EQUATION USING FLUX-FREE ERROR ESTIMATES *
}

\author{
NÚRIA PARÉS, PEDRO DÍEZ AND ANTONIO HUERTA †
}

\begin{abstract}
The paper introduces a methodology to compute strict upper and lower bounds for linear-functional outputs of the exact solutions of the advection-reaction-diffusion equation. The proposed approach is an alternative to the standard residual type estimators (hybrid-flux), circumventing the need of flux-equilibration following a fluxfree error estimation strategy. The presented estimator provides sharper estimates than the ones provided by both the standard hybrid-flux techniques and other flux-free techniques.
\end{abstract}

Key words. Linear-functional outputs; Exact/guaranteed/strict bounds; Error estimation; Goal-oriented adaptivity; Advection-reaction-diffusion equation.

AMS subject classifications. 65N15, 65N30, 49M29

1. Introduction. A posteriori error estimation techniques for the advection-reactiondiffusion equation were first introduced in the 1980s [27, 33, 31]. These pioneering error estimates are global in nature, providing bounds of the approximation error in energy-type norms defined over the entire domain. It was not until the late 1990s when strategies to compute bounds for linear functional outputs of the solution where developed [19, 18, 26, 4, 10]. These methods, based on duality techniques provide error bounds of local quantities of interest, outputs here-in.

The strategies providing two-sided bounds of the error in a demanded output rely on estimating the error in global energy-type norms. The error estimate for the output is obtained combining energy estimates associated to the original problem and to an auxiliary adjoint problem. In particular, for the non-symmetric advection-diffusion-reaction model problem, the energy estimates are required for some symmetrized self-adjoint error problems (diffusion-reaction problems).

For advection dominated problems, the use of stabilized formulations [9] is of outmost importance. In the present paper we restrict ourselves to standard (non-stabilized) formulations. The use of similar techniques for stabilized methods is going to be presented in a forthcoming paper.

Any strategy providing energy error estimates for the symmetrized diffusion-reaction problems may be used to produce estimates for the output. A posteriori error estimates in global energy norms for the reaction-diffusion equation have been the subject of intense research activity, see for instance [35, 1, 37, 36, 28, 11]. Note, however, that not all the a posteriori estimates provide strict bounds. For instance, in some cases the expressions that bound the error involve continuity or interpolation constants which have to be approximated. Obviously, the cost of guaranteeing strict bounds is in general larger than the cost of the estimates such that their bounding properties depend on the proper evaluation of unknown constants.

In the present work, the energy error estimates for the diffusion-reaction problems are obtained using implicit residual error estimators. These estimators are based on approximating the error equation by solving local problems. The main ingredients for this kind of error estimators are the domain decomposition technique and the method to solve the local

\footnotetext{
*This work was partially supported by Ministerio de Educación y Ciencia, Grants DPI2007-62395 and BIA200766965 and by Generalitat de Catalunya AGAUR, Grant 2005 SGR917.

† Laboratori de Càlcul Numèric (LaCàN), Universitat Politècnica de Catalunya, C/Jordi Girona 1-3 E-08034 Barcelona, Spain (e-mail: \{nuria.pares, pedro.diez, antonio.huerta\}@upc.edu).
} 
error problem. The domain decomposition pertains to the selection of the local subdomains where the error equation is solved and, eventually, to the local boundary conditions required to obtain well-posed local problems. Basically, two options are available for the domain decomposition: 1) the hybrid-flux techniques that use elements as subdomains and require obtaining equilibrated fluxes around the elements as boundary conditions for the local error problems and 2) the flux-free techniques where the subdomains are the patches of elements around every vertex node of the mesh.

The hybrid flux idea $[13,2]$ is the basis of the classical implicit residual estimates. Previous to solving the local problems the hybrid fluxes have to be computed using a fluxequilibration complex algorithm, strongly dependent on the element type and requiring a data structure that is not natural in a standard finite element code.

The flux-free approach is a promising alternative to standard implicit residual error estimators precluding the necessity of computing equilibrated hybrid fluxes $[22,6,15,16]$. The size of the subdomains where the local problems have to be solved is larger than for the hybrid flux case, each patch (also called star) containing several elements of the computational mesh. However, the number of local problems to solve is much lower: recall that in a typical $3 \mathrm{D}$ tetrahedral mesh, the ratio elements/vertex nodes is equal to 6. Moreover, the main advantage of these strategies is precisely their flux-free character, that is the boundary conditions are natural and hence their implementation is straightforward. The domain decomposition is performed using a partition of unity strategy. The resulting estimates are hence much simpler from the implementation viewpoint, especially in the 3D cases, and provide upper bounds of the error (as well as the standard implicit residual estimators with equilibration of hybrid fluxes).

In the first papers introducing the flux-free idea, the local problems arising from the fluxfree domain decomposition strategy are solved using a finite element mesh inside each local subdomain. Consequently, the resulting estimates are said to be asymptotic error bounds, that is with respect to a reference solution associated with a finer reference mesh. This may be not satisfactory enough, specially if the computational mesh is coarse and the quality of the reference solution is not guaranteed to be accurate enough. The actual paradigm is to produce estimates providing bounds with respect to the exact solution, without any further assumption on a truth reference mesh.

Techniques to obtain exact bounds have been developed in the hybrid flux framework $[29,30,21,39,23,24]$. The idea is simply solving the local problem using a dual formulation and to minimize the complementary energy. In the present paper this approach is extended to the flux-free technique presented in [22]. Thus, exact bounds are recovered without the need of computing local hybrid fluxes and precluding the annoying implementation of the equilibration techniques. In fact, the resulting estimates have similar features as their asymptotic version, while providing a guaranteed upper bound.

The strategy presented here is also applicable to different flux-free estimates, see for instance $[6,15,16]$. However, following the ideas presented in [22] the resulting estimates are much sharper in the asymptotic version and they are therefore preferred to be extended. This behavior is also observed in [32], where the approach presented in [22] is also compared to other flux-free techniques in the context of the generalized finite element method.

2. Problem statement. Consider the steady advection-reaction-diffusion equation posed on a polygonal domain $\Omega$. The boundary $\partial \Omega$ is divided into two disjoint parts $\Gamma_{\mathrm{D}}$ and $\Gamma_{\mathrm{N}}$ such that $\bar{\Gamma}_{\mathrm{D}} \cup \bar{\Gamma}_{\mathrm{N}}=\partial \Omega, \Gamma_{\mathrm{D}} \cap \Gamma_{\mathrm{N}}=\emptyset$ and $\Gamma_{\mathrm{D}}$ is a non empty set. The weak solution of the problem is $u \in \mathcal{U}$ verifying

$$
a(u, v)=\ell(v) \quad \forall v \in \mathcal{V},
$$


where

$$
a(w, v):=\int_{\Omega}[\nu \nabla w \cdot \nabla v+(\boldsymbol{\alpha} \cdot \nabla w) v+\sigma w v] \mathrm{d} \Omega, \quad \ell(v):=\int_{\Omega} f v \mathrm{~d} \Omega+\int_{\Gamma_{\mathrm{N}}} g v \mathrm{~d} \Gamma .
$$

The solution and test spaces are $\mathcal{U}:=\left\{v \in \mathcal{H}^{1}(\Omega),\left.v\right|_{\Gamma_{\mathrm{D}}}=u_{\mathrm{D}}\right\}$ and $\mathcal{V}:=\left\{v \in \mathcal{H}^{1}(\Omega)\right.$, $\left.\left.v\right|_{\Gamma_{\mathrm{D}}}=0\right\}$ respectively where $\mathcal{H}^{1}$ is the standard Sobolev space of square integrable functions whose first derivative is also square integrable. The data is assumed to be smooth, that is, $f \in \mathcal{H}^{-1}(\Omega), g \in \mathcal{H}^{-\frac{1}{2}}\left(\Gamma_{\mathrm{N}}\right), u_{\mathrm{D}} \in \mathcal{H}^{\frac{1}{2}}\left(\Gamma_{\mathrm{D}}\right), \nu \in \mathcal{L}^{\infty}(\Omega), \sigma \in \mathcal{L}^{\infty}(\Omega)$ is a non-negative real coefficient and the prescribed vector field $\alpha \in \mathcal{H}(\operatorname{div} ; \Omega)$. Additionally, the nonsymmetric bilinear form $a(\cdot, \cdot)$ is assumed to be continuous and coercive in $\mathcal{V}$, and therefore it is assumed that $\sigma-\frac{1}{2} \boldsymbol{\nabla} \cdot \boldsymbol{\alpha} \geq 0$ in $\Omega$ and also that the Dirichlet boundary contains the inflow boundaries, that is $\Gamma^{-} \subset \Gamma_{\mathrm{D}}$ for $\Gamma^{-}:=\{\boldsymbol{x} \in \partial \Omega, \boldsymbol{\alpha} \cdot \boldsymbol{n}<0\}$.

The goal of the vast majority of finite element simulations is to determine specific quantities (outputs) which depend on the solution of the partial differential equations governing the problem. In most cases, moreover, it is crucial to be able to certify the precision of the approximations of these outputs. Therefore the final goal of most simulations is to provide upper and lower bounds for the exact value of the quantity of interest, or equivalently, to provide a range where the exact value of the output lies.

Here, the quantities of interest are restricted to depend linearly on $u$ and to be of the form

$$
\ell^{\mathcal{O}}(u):=\int_{\Omega} f^{\mathcal{O}} u \mathrm{~d} \Omega+\int_{\Gamma_{\mathrm{N}}} g^{\mathcal{O}} u \mathrm{~d} \Gamma
$$

where $f^{\mathcal{O}} \in \mathcal{H}^{-1}(\Omega)$ and $g^{\mathcal{O}} \in \mathcal{H}^{-\frac{1}{2}}\left(\Gamma_{\mathrm{N}}\right)$. The goal is then to provide upper and lower bounds for $s=\ell^{\mathcal{O}}(u)$, namely,

$$
s^{l b} \leq s \leq s^{u b}
$$

and at the same time derive an adaptive refinement procedure to be able to improve the accuracy of the desired output (i.e. narrow the gap between the bounds $s^{u b}-s^{l b}$ ).

REMARK 1. The form of the output (2.2) includes weighed averages of the solution $u$ over a region of $\Omega$ and weighted averages of $u$ over a curve in a portion of $\Gamma_{\mathrm{N}}$. However, the integral of a weighted flux over a portion of $\Gamma_{\mathrm{D}}$ and the integral of the gradient of $u$ times a weighting function over a region of $\Omega$ may also be rewritten in the form (2.2). That is, equation (2.2) implicitly contains outputs of the form

$$
\ell^{\mathcal{O}}(u)=\int_{\Gamma_{\mathrm{D}}} \omega_{1}^{\mathcal{O}} \nabla u \cdot \boldsymbol{n} \mathrm{d} \Gamma+\int_{\Omega} \omega_{2}^{\mathcal{O}} \nabla u \cdot \nabla \chi_{2} \mathrm{~d} \Omega
$$

where $\omega_{1}^{\mathcal{O}}, \omega_{2}^{\mathcal{O}}$ and $\chi_{2}$ are appropriate functions, see reference [30]. This restriction in the form of the linear-functional output is due to the fact that the present approach aims at obtaining strict bounds for the quantity of interest - see equation (5.3) in section 5. If only an approximation or asymptotic bounds are required, the quantity of interest may take a more general form.

3. Energy reformulation: representation of the output bounds. Most existing techniques to obtain upper and lower bounds for a quantity of interest $\ell^{\mathcal{O}}(u)$ are based on the fact that bounds for the output may be obtained using available techniques for estimating the error measured in the energy norm, see for instance [19, 17, 2, 3]. The key point of these strategies is to recover an alternative representation for the output $\ell^{\mathcal{O}}(u)$ (or in the case of non-selfadjoint problems for its bounds) in terms of the energy norms of some continuous 
functions. This alternative representation does not directly yield a computable expression for the bounds of the output because the energy norms appearing in the expression are noncomputable. However, bounds may be easily recovered using existing strategies providing upper and lower bounds for the error measured in the energy norm.

This section is devoted to describe in detail the representation of bounds for $\ell^{\mathcal{O}}(u)$ in terms of energy norms for the case of the advection-reaction-diffusion equation which is non-selfadjoint, see [19]. That is, the key ingredients of the methods to obtain bounds for $s=\ell^{\mathcal{O}}(u)$ from upper bounds for the energy norm are briefly summarized.

The non-computable expressions for the upper and lower bounds for $\ell^{\mathcal{O}}(u)$ are recovered in three steps. First, the finite element approximation of $u, u_{h}$, is computed. This approximation is associated with a finite element mesh of the domain $\Omega$ and with a finite element interpolation space $\mathcal{U}^{h} \subset \mathcal{U}$, that is $u_{h} \in \mathcal{U}^{h}$. Second, an adjoint problem associated with the selected output is introduced, along with its finite element approximation, $\psi_{h} \in \mathcal{V}^{h} \subset \mathcal{V}$. Finally, the error equations for $u_{h}$ and $\psi_{h}$ are modified (symmetrizing the 1.h.s.) such that bounds of $s=\ell^{\mathcal{O}}(u)$ are obtained from linear combinations of the resulting modified errors.

Indeed, let $\mathcal{U}^{h} \subset \mathcal{U}$ and $\mathcal{V}^{h} \subset \mathcal{V}$ be the finite element interpolation spaces associated with a finite element mesh of characteristic size $h$ and degree $p$ for the complete polynomial base. Then, the bounding procedure may be sketched as:

1. Compute the finite element approximation of the primal problem: find $u_{h} \in \mathcal{U}^{h}$ such that

$$
a\left(u_{h}, v\right)=\ell(v) \quad \forall v \in \mathcal{V}^{h}
$$

2. Introduce the adjoint problem associated with the selected output: find $\psi \in \mathcal{V}$ such that

$$
a(v, \psi)=\ell^{\mathcal{O}}(v) \quad \forall v \in \mathcal{V},
$$

and compute its finite element approximation: find $\psi_{h} \in \mathcal{V}^{h}$ such that

$$
a\left(v, \psi_{h}\right)=\ell^{\mathcal{O}}(v) \quad \forall v \in \mathcal{V}^{h} .
$$

3. Recover the bounds for the output in the three following steps:

3.1. Consider the errors in the approximations $u_{h}$ and $\psi_{h}, e:=u-u_{h} \in \mathcal{V}$ and $\varepsilon:=\psi-\psi_{h} \in \mathcal{V}$, satisfying the residual equations

$$
a(e, v)=\ell(v)-a\left(u_{h}, v\right)=: R^{\mathrm{P}}(v) \quad \forall v \in \mathcal{V},
$$

and

$$
a(v, \varepsilon)=\ell^{\mathcal{O}}(v)-a\left(v, \psi_{h}\right)=: R^{\mathrm{D}}(v) \quad \forall v \in \mathcal{V},
$$

where $R^{\mathrm{P}}(\cdot)$ and $R^{\mathrm{D}}(\cdot)$ stand for the weak primal and adjoint residuals associated with the approximations $u_{h}$ and $\psi_{h}$ respectively.

3.2. Introduce the modified symmetric versions of the residual problems: find $e^{\mathrm{s}}$ and $\varepsilon^{\mathrm{s}} \in \mathcal{V}$ such that

$$
a^{\mathrm{s}}\left(e^{\mathrm{s}}, v\right)=R^{\mathrm{P}}(v) \quad \forall v \in \mathcal{V}
$$

and

$$
a^{\mathrm{s}}\left(\varepsilon^{\mathrm{s}}, v\right)=R^{\mathrm{D}}(v) \quad \forall v \in \mathcal{V}
$$


where $a^{\mathrm{S}}(\cdot, \cdot)$ is the symmetric counterpart of $a(\cdot, \cdot)$

$$
\begin{aligned}
a^{\mathrm{s}}(w, v) & =\frac{1}{2}(a(w, v)+a(v, w)) \\
& =\int_{\Omega}\left[\nu \nabla w \cdot \nabla v+\left(\sigma-\frac{1}{2} \boldsymbol{\nabla} \cdot \boldsymbol{\alpha}\right) w v\right] \mathrm{d} \Omega+\frac{1}{2} \int_{\Gamma_{\mathrm{N}}} \boldsymbol{\alpha} \cdot \boldsymbol{n} w v \mathrm{~d} \Gamma
\end{aligned}
$$

3.3. Compute the upper and lower bounds for $s$ as

$s^{l b}:=\ell^{\mathcal{O}}\left(u_{h}\right)-\frac{1}{4}\left\|\kappa e^{\mathrm{s}}-\frac{1}{\kappa} \varepsilon^{\mathrm{s}}\right\|_{u b}^{2} \leq \ell^{\mathcal{O}}(u) \leq \ell^{\mathcal{O}}\left(u_{h}\right)+\frac{1}{4}\left\|\kappa e^{\mathrm{s}}+\frac{1}{\kappa} \varepsilon^{\mathrm{s}}\right\|_{u b}^{2}=: s^{u b}$,

where $\|\cdot\|$ is the energy norm induced by the bilinear form $a^{\mathrm{s}}(\cdot, \cdot),\|v\|^{2}=$ $a^{\mathrm{s}}(v, v)=a(v, v),\|v\|_{u b}$ represents an upper bound for the value of $\|v\|$ and $\kappa \in \mathbb{R}$ is an arbitrary scalar non-zero parameter.

The following theorem shows that the strategy sketched above provides effective bounds for $s$.

THEOREM 3.1. Let $e^{\mathrm{s}}$ and $\varepsilon^{\mathrm{s}} \in \mathcal{V}$ be such that for any $v \in \mathcal{V}$

$$
a^{\mathrm{s}}\left(e^{\mathrm{s}}, v\right)=R^{P}(v) \quad \text { and } \quad a^{\mathrm{s}}\left(\varepsilon^{\mathrm{s}}, v\right)=R^{D}(v)
$$

Then, the following inequality holds for the exact values of $e^{\mathrm{s}}$ and $\varepsilon^{\mathrm{s}}$

$$
\ell^{\mathcal{O}}\left(u_{h}\right)-\frac{1}{4}\left\|\kappa e^{\mathrm{s}}-\frac{1}{\kappa} \varepsilon^{\mathrm{s}}\right\|^{2} \leq \ell^{\mathcal{O}}(u) \leq \ell^{\mathcal{O}}\left(u_{h}\right)+\frac{1}{4}\left\|\kappa e^{\mathrm{s}}+\frac{1}{\kappa} \varepsilon^{\mathrm{s}}\right\|^{2},
$$

and therefore, if energy estimates giving upper bounds for $\left\|\kappa e^{\mathrm{s}}-\frac{1}{\kappa} \varepsilon^{\mathrm{s}}\right\|$ are available, one gets

$$
\ell^{\mathcal{O}}\left(u_{h}\right)-\frac{1}{4}\left\|\kappa e^{\mathrm{s}}-\frac{1}{\kappa} \varepsilon^{\mathrm{s}}\right\|_{u b}^{2} \leq \ell^{\mathcal{O}}(u) \leq \ell^{\mathcal{O}}\left(u_{h}\right)+\frac{1}{4}\left\|\kappa e^{\mathrm{s}}+\frac{1}{\kappa} \varepsilon^{\mathrm{s}}\right\|_{u b}^{2} .
$$

Proof. Taking $v=e$ in (3.2) and using $a\left(e, \psi_{h}\right)=0$ (Galerkin orthogonality) yields the following error representation

$$
R^{\mathrm{D}}(e)=\ell^{\mathcal{O}}(e)-a\left(e, \psi_{h}\right)=\ell^{\mathcal{O}}(e)=\ell^{\mathcal{O}}\left(u-u_{h}\right)=\ell^{\mathcal{O}}(u)-\ell^{\mathcal{O}}\left(u_{h}\right) .
$$

Similarly, taking $v=e$ in equation (3.1) yields

$$
R^{\mathrm{P}}(e)=a(e, e)=\|e\|^{2} .
$$

The proof now follows from a simple algebraic manipulation. For any scalar $\kappa \neq 0$, we have that

$$
\begin{aligned}
0 \leq\left\|\frac{1}{2}\left(\kappa e^{\mathrm{s}} \pm \frac{1}{\kappa} \varepsilon^{\mathrm{s}}\right)-\kappa e\right\|^{2} & =\frac{1}{4}\left\|\kappa e^{\mathrm{s}} \pm \frac{1}{\kappa} \varepsilon^{\mathrm{s}}\right\|^{2}+\kappa^{2}\|e\|^{2}-\kappa a^{\mathrm{s}}\left(\kappa e^{\mathrm{s}} \pm \frac{1}{\kappa} \varepsilon^{\mathrm{s}}, e\right) \\
& =\frac{1}{4}\left\|\kappa e^{\mathrm{s}} \pm \frac{1}{\kappa} \varepsilon^{\mathrm{s}}\right\|^{2}+\kappa^{2}\|e\|^{2}-\kappa^{2} a^{\mathrm{s}}\left(e^{\mathrm{s}}, e\right) \mp a^{\mathrm{s}}\left(\varepsilon^{\mathrm{s}}, e\right) \\
& =\frac{1}{4}\left\|\kappa e^{\mathrm{s}} \pm \frac{1}{\kappa} \varepsilon^{\mathrm{s}}\right\|^{2}+\kappa^{2}\|e\|^{2}-\kappa^{2} R^{\mathrm{P}}(e) \mp R^{\mathrm{D}}(e) \\
& =\frac{1}{4}\left\|\kappa e^{\mathrm{s}} \pm \frac{1}{\kappa} \varepsilon^{\mathrm{s}}\right\|^{2}+\kappa^{2}\|e\|^{2}-\kappa^{2}\|e\|^{2} \mp\left(\ell^{\mathcal{O}}(u)-\ell^{\mathcal{O}}\left(u_{h}\right)\right) \\
& = \pm \ell^{\mathcal{O}}\left(u_{h}\right)+\frac{1}{4}\left\|\kappa e^{\mathrm{s}} \pm \frac{1}{\kappa} \varepsilon^{\mathrm{s}}\right\|^{2} \mp \ell^{\mathcal{O}}(u),
\end{aligned}
$$


where equations (3.3), (3.4), (3.7) and (3.8) have been used. Consequently,

$$
0 \leq\left\|\frac{1}{2}\left(\kappa e^{\mathrm{s}} \pm \frac{1}{\kappa} \varepsilon^{\mathrm{s}}\right)-\kappa e\right\|^{2}= \pm \ell^{\mathcal{O}}\left(u_{h}\right)+\frac{1}{4}\left\|\kappa e^{\mathrm{s}} \pm \frac{1}{\kappa} \varepsilon^{\mathrm{s}}\right\|^{2} \mp \ell^{\mathcal{O}}(u),
$$

that is

$$
\pm \ell^{\mathcal{O}}(u) \leq \pm \ell^{\mathcal{O}}\left(u_{h}\right)+\frac{1}{4}\left\|\kappa e^{\mathrm{s}} \pm \frac{1}{\kappa} \varepsilon^{\mathrm{s}}\right\|^{2} .
$$

The proof is concluded by noting that the + sign in the previous equality yields the expression for the upper bound of $\ell^{\mathcal{O}}(u)$, whereas the - sign yields the expression for the lower bound of $\ell^{\mathcal{O}}(u)$.

The next section introduces a methodology to obtain strict upper bounds for the energy norm. This approach is then used to compute $\left\|\kappa e^{\mathrm{s}} \pm 1 / \kappa \varepsilon^{\mathrm{s}}\right\|_{u b}^{2}$. Note that, in the following, the subscript $u b$ in the norm is just a notation to denote an upper bound estimate: $\|\cdot\|_{u b}$ is not intending to be an alternative norm different than $\|\cdot\|$. Thus, for a concrete function $v,\|v\|_{u b}$ is an overestimated approximation of $\|v\|$.

4. Upper bounds for the energy norm: complementary energy relaxation. Consider the auxiliary function $z \in \mathcal{V}$ solution of

$$
a^{\mathrm{s}}(z, v)=R^{*}(v) \quad \forall v \in \mathcal{V},
$$

where $R^{*}(v)=\alpha R^{\mathrm{P}}(v)+\beta R^{\mathrm{D}}(v)$ for $\alpha, \beta \in \mathbb{R}$. Note that for $\alpha=1$ and $\beta=0, R^{*}(v)=$ $R^{\mathrm{P}}(v)$ and problem (4.1) is the residual problem for $e^{\mathrm{s}}$. Therefore in this case $z=e^{\mathrm{s}}$. Analogously, the choice of $\alpha=0$ and $\beta=1$, produces $R^{*}(v)=R^{\mathrm{D}}(v)$ and the residual problem for $\varepsilon^{\mathrm{s}}$ is recovered yielding $z=\varepsilon^{\mathrm{s}}$. In particular, $\alpha=\kappa$ and $\beta= \pm 1 / \kappa$ will be used later to obtain the required upper bounds for $\left\|\kappa e^{\mathrm{s}} \pm 1 / \kappa \varepsilon^{\mathrm{s}}\right\|^{2}$.

The purpose of this section is to establish a procedure to compute upper bounds of $\|z\|^{2}$. Recently, much effort has been devoted to obtain exact bounds, that is bounds guaranteed with respect to the exact solution independently of any underlying mesh (see the series of references [29, 30, 21, 39, 23, 24]). All these strategies recover strict bounds for $\|z\|$ using the standard complementary energy approach. The key idea is to relax the continuous problem of finding $z \in \mathcal{V}$ fulfilling equation (4.1). The resulting problem is equivalent to the original one but expressed in terms of dual unknowns living in larger spaces with less regularity requirements. Other similar strategies providing bounds, also based on a dual formulation, have been used by other authors [7, 14, 28, 11, 38, 5].

The relaxed problem consists in obtaining a pair of dual estimates $\hat{\boldsymbol{q}} \in\left[\mathcal{L}^{2}(\Omega)\right]^{2}$ and $\hat{r} \in \mathcal{L}^{2}(\Omega)$ such that

$$
\int_{\Omega}\left[\nu \hat{\boldsymbol{q}} \cdot \boldsymbol{\nabla} v+\left(\sigma-\frac{1}{2} \boldsymbol{\nabla} \cdot \boldsymbol{\alpha}\right) \hat{r} v\right] \mathrm{d} \Omega+\frac{1}{2} \int_{\Gamma_{\mathrm{N}}} \boldsymbol{\alpha} \cdot \boldsymbol{n} \hat{r} v \mathrm{~d} \Gamma=a^{\mathrm{s}}(z, v)=R^{*}(v) \quad \forall v \in \mathcal{V} .
$$

Any pair of dual estimates $\hat{\boldsymbol{q}}$ and $\hat{r}$ verifying the previous equation yield an upper bound for $\|z\|$. This is stated in the following theorem. In the remainder, for the sake of a simpler notation, the coefficient in the reaction term is denoted by $\tilde{\sigma}$, namely $\tilde{\sigma}=\sigma-\frac{1}{2} \boldsymbol{\nabla} \cdot \boldsymbol{\alpha}$.

THEOREM 4.1. Let $\hat{\boldsymbol{q}} \in\left[\mathcal{L}^{2}(\Omega)\right]^{2}$ and $\hat{r} \in \mathcal{L}^{2}(\Omega)$ fulfill equation (4.2). Then, an upper bound for the energy norm of the solution $z$ of (4.1) is computed as

$$
\|z\|^{2} \leq \int_{\Omega}\left[\nu \hat{\boldsymbol{q}} \cdot \hat{\boldsymbol{q}}+\tilde{\sigma} \hat{r}^{2}\right] \mathrm{d} \Omega+\frac{1}{2} \int_{\Gamma_{\mathrm{N}}} \boldsymbol{\alpha} \cdot \boldsymbol{n} \hat{r}^{2} \mathrm{~d} \Gamma .
$$


Proof. The result follows after the following algebraic manipulation

$$
\begin{aligned}
& 0 \leq \int_{\Omega}\left[\nu(\hat{\boldsymbol{q}}-\boldsymbol{\nabla} z) \cdot(\hat{\boldsymbol{q}}-\boldsymbol{\nabla} z)+\tilde{\sigma}(\hat{r}-z)^{2}\right] \mathrm{d} \Omega+\frac{1}{2} \int_{\Gamma_{\mathrm{N}}} \boldsymbol{\alpha} \cdot \boldsymbol{n}(\hat{r}-z)^{2} \mathrm{~d} \Gamma \\
& =\int_{\Omega}\left[\nu \hat{\boldsymbol{q}} \cdot \hat{\boldsymbol{q}}+\tilde{\sigma} \hat{r}^{2}\right] \mathrm{d} \Omega+\frac{1}{2} \int_{\Gamma_{\mathrm{N}}} \boldsymbol{\alpha} \cdot \boldsymbol{n} \hat{r}^{2} \mathrm{~d} \Gamma \\
& +\int_{\Omega}\left[\nu \nabla z \cdot \nabla z+\tilde{\sigma} z^{2}\right] \mathrm{d} \Omega+\frac{1}{2} \int_{\Gamma_{\mathrm{N}}} \boldsymbol{\alpha} \cdot \boldsymbol{n} z^{2} \mathrm{~d} \Gamma \\
& -2\left(\int_{\Omega}[\nu \hat{\boldsymbol{q}} \cdot \nabla z+\tilde{\sigma} \hat{r} z] \mathrm{d} \Omega+\frac{1}{2} \int_{\Gamma_{\mathrm{N}}} \boldsymbol{\alpha} \cdot \boldsymbol{n} \hat{r} z \mathrm{~d} \Gamma\right) \\
& =\int_{\Omega}\left[\nu \hat{\boldsymbol{q}} \cdot \hat{\boldsymbol{q}}+\tilde{\sigma} \hat{r}^{2}\right] \mathrm{d} \Omega+\frac{1}{2} \int_{\Gamma_{\mathrm{N}}} \boldsymbol{\alpha} \cdot \boldsymbol{n} \hat{r}^{2} \mathrm{~d} \Gamma+a^{\mathrm{s}}(z, z)-2 a^{\mathrm{s}}(z, z) \\
& =\int_{\Omega}\left[\nu \hat{\boldsymbol{q}} \cdot \hat{\boldsymbol{q}}+\tilde{\sigma} \hat{r}^{2}\right] \mathrm{d} \Omega+\frac{1}{2} \int_{\Gamma_{\mathrm{N}}} \boldsymbol{\alpha} \cdot \boldsymbol{n} \hat{r}^{2} \mathrm{~d} \Gamma-\|z\|^{2},
\end{aligned}
$$

where both equation (4.2) with $v=z$ and the definition of the bilinear form $a^{\mathrm{s}}(\cdot, \cdot)$, equation (3.5) with $w=v=z$, are used. $\square$

REMARK 2. In the frequent case of an incompressible prescribed vector field $\boldsymbol{\alpha}$, i.e. $\boldsymbol{\nabla} \cdot \boldsymbol{\alpha}=0$, if either only Dirichlet boundary conditions are prescribed or $\left.\boldsymbol{\alpha} \cdot \boldsymbol{n}\right|_{\Gamma_{\mathrm{N}}}=0$, bounds for $\|z\|$ are obtained using the simplified expression

$$
\|z\|^{2} \leq \int_{\Omega}\left[\nu \hat{\boldsymbol{q}} \cdot \hat{\boldsymbol{q}}+\sigma \hat{r}^{2}\right] \mathrm{d} \Omega .
$$

REMARK 3. Note that the relaxed problem (4.2) admits at least a trivial solution $\hat{\boldsymbol{q}}=$ $\nabla z \in\left[\mathcal{L}^{2}(\Omega)\right]^{2}$ and $\hat{r}=z \in \mathcal{L}^{2}(\Omega)$. In this case, expression (4.3) turns into an equality. Any other values of $\hat{\boldsymbol{q}}$ and $\hat{r}$ fulfilling equation (4.2) yield upper bounds for $\|z\|^{2}$. In order to obtain sharper values for the upper bound, $\hat{\boldsymbol{q}}$ and $\hat{r}$ have to be good approximations of $\nabla z$ and $z$ respectively.

Theorem 4.1 allows to compute strict upper bounds for $\|z\|$ recovering two globally equilibrated dual estimates $\hat{\boldsymbol{q}}$ and $\hat{r}$, i.e. verifying equation (4.2). However, the essential feature of the method is that if the fields $f, g, f^{\mathcal{O}}$ and $g^{\mathcal{O}}$ are piecewise polynomial fields, it is possible to determine - amongst all the dual estimates $\hat{\boldsymbol{q}} \in\left[\mathcal{L}^{2}(\Omega)\right]^{2}$ and $\hat{r} \in \mathcal{L}^{2}(\Omega)$ verifying equation (4.2) - two piecewise polynomial fields verifying equation (4.2). That is, for a given suitable interpolation degree $q$, it is possible to find $\hat{\boldsymbol{q}} \in\left[\widehat{\mathbb{P}}^{q}(\Omega)\right]^{2}$ and $\hat{r} \in \widehat{\mathbb{P}}^{q}(\Omega)$ verifying equation (4.2) where

$$
\widehat{\mathbb{P}}^{q}(\Omega):=\left\{v \in \mathcal{L}^{2}(\Omega),\left.v\right|_{\Omega_{k}} \in \mathbb{P}^{q}\left(\Omega_{k}\right)\right\}
$$

Here a triangulation of the computational domain $\Omega$ into $n_{\mathrm{el}}$ triangles is considered where $\Omega_{k}$ denote a general triangle, $k=1, \ldots, n_{\mathrm{el}}$.

REMARK 4. A proof of the existence of a piecewise polynomial solution $\hat{\boldsymbol{q}} \in\left[\widehat{\mathbb{P}}^{q}(\Omega)\right]^{2}$ and $\hat{r} \in \widehat{\mathbb{P}}^{q}(\Omega)$ of equation (4.2) may be found in [29,30]. Note that if one chooses $\hat{r}=0$, the existence of a piecewise polynomial dual estimate $\hat{\boldsymbol{q}}$ verifying

$$
\int_{\Omega} \nu \hat{\boldsymbol{q}} \cdot \nabla v \mathrm{~d} \Omega=R^{*}(v) \quad \forall v \in \mathcal{V}
$$

may be related to the decomposition of the space $\mathcal{H}\left(\right.$ div,$\left.\Omega_{k}\right)$ used in the context of mixed or hybrid elements, see [25]. 
Hence, the computation of strict upper bounds for $\|z\|$ is reduced to a discrete problem: find $\hat{\boldsymbol{q}} \in\left[\widehat{\mathbb{P}}^{q}(\Omega)\right]^{2}$ and $\hat{r} \in \widehat{\mathbb{P}}^{q}(\Omega)$ verifying equation (4.2). At first sight, this problem is discrete (with finite number of d.o.f.) but global, that is, affecting the whole domain $\Omega$. Thankfully, proper domain decomposition techniques allow decomposing the global discrete problem into local problems. That is, the piecewise polynomial fields $\hat{\boldsymbol{q}}$ and $\hat{r}$ are to be computed solving local discrete problems.

In [30], the dual estimates $\hat{\boldsymbol{q}}$ and $\hat{r}$ result of solving local independent problems in each element of the mesh. This requires the use of flux-equilibration techniques (hybrid-flux techniques) to properly set the boundary conditions for the local elementary problems. Following this approach, first, the equilibrated residual method is used to compute the equilibrated fluxes at the interelementary edges of the mesh. These fluxes are then used as local boundary conditions to compute the dual estimates $\hat{\boldsymbol{q}}$ and $\hat{r}$ in each triangle of the mesh.

The next section presents a new approach to compute the dual estimates $\hat{\boldsymbol{q}}$ and $\hat{r}$. The idea is to avoid the use of flux-equilibration techniques and, instead, use the flux-free estimator proposed in [22]. This domain decomposition technique exploits the partition-of-unity property to reduce the problems from $\Omega$ to subdomains different than elements. The local problems for the dual estimates $\hat{\boldsymbol{q}}$ and $\hat{r}$ are posed over patches of elements. The advantage of this approach is that the local problems are naturally equilibrated and do not require enforcing equilibrium.

As mentioned before, the strategy presented here only provides strict bounds for problems where the input data are piecewise polynomial. However, using the strategies presented in $[34,12]$ this restriction on the fields $f, g, f^{\mathcal{O}}$ and $g^{\mathcal{O}}$ could be removed. This requires to consider non polynomial representations of $\hat{\boldsymbol{q}}$ and $\hat{r}$ in the triangles of the mesh, which is more complex and less natural to implement. Thus, in order to deal with non polynomial data, the local polynomial representation of $\hat{\boldsymbol{q}}$ and $\hat{r}$ is replaced by the local construction given in [34], only in those elements where the source term or boundary conditions are non polynomial.

5. Local computation of the dual estimates $\hat{\boldsymbol{q}}$ and $\hat{r}$ using a flux-free approach. This section is devoted to detail the computation of the piecewise polynomial dual estimates $\hat{\boldsymbol{q}}$ and $\hat{r}$ using the flux-free approach proposed in [22]. After introducing some notation, the domain decomposition strategy used to localize the computation of $\hat{\boldsymbol{q}}$ and $\hat{r}$ is presented. The solvability of the local problems is discussed along with the verification that the dual estimates $\hat{\boldsymbol{q}}$ and $\hat{r}$ verify equation (4.2). Finally some computational aspects are discussed.

5.1. Domain decomposition. Let $\boldsymbol{x}^{i} i=1, \ldots, n_{\mathrm{np}}$ denote the vertices of the elements (triangles) in the computational mesh (thus linked to $\mathcal{U}^{h}$ ) and $\phi^{i}$ denote the corresponding linear shape functions, which are such that $\phi^{i}\left(\boldsymbol{x}^{j}\right)=\delta_{i j}$. The support of $\phi^{i}$ is denoted by $\omega^{i}$ and is called the star centered in/associated with vertex $\boldsymbol{x}^{i}$. It is important to recall that the linear shape functions based on the vertices are a partition of unity, namely

$$
\sum_{i=1}^{n_{\mathrm{np}}} \phi^{i}=1 .
$$

Let also $\mathcal{V}\left(\omega^{i}\right)$ and $\widehat{\mathbb{P}}^{q}\left(\omega^{i}\right)$ denote the local restrictions of the spaces $\mathcal{V}$ and $\widehat{\mathbb{P}}^{q}(\Omega)$ to the star $\omega^{i}$. Formally, any function $v \in \mathcal{V}\left(\omega^{i}\right)$ or $v \in \widehat{\mathbb{P}}^{q}\left(\omega^{i}\right)$ is not defined on the whole domain $\Omega$ but only on the star $\omega^{i}$. However, here any $v \in \mathcal{V}\left(\omega^{i}\right)$ or $v \in \widehat{\mathbb{P}}^{q}\left(\omega^{i}\right)$ is naturally extended to $\Omega$ by setting the values outside $\omega^{i}$ to zero. Thus, functions in $\mathcal{V}\left(\omega^{i}\right)$ are continuous in $\omega^{i}$ but generally discontinuous across the boundary of the star $\omega^{i}$, whereas functions in $\widehat{\mathbb{P}}^{q}\left(\omega^{i}\right)$ are piecewise polynomial functions in the triangles contained in $\omega^{i}$ vanishing on the elements outside $\omega^{i}$. 
The dual estimates $\hat{\boldsymbol{q}}$ and $\hat{r}$ are decomposed as

$$
\hat{\boldsymbol{q}}=\sum_{i=1}^{n_{\mathrm{np}}} \hat{\boldsymbol{q}}^{i} \quad \text { and } \quad \hat{r}=\sum_{i=1}^{n_{\mathrm{np}}} \hat{r}^{i}
$$

where the local estimates $\hat{\boldsymbol{q}}^{i} \in\left[\widehat{\mathbb{P}}^{q}\left(\omega^{i}\right)\right]^{2}$ and $\hat{r}^{i} \in \widehat{\mathbb{P}}^{q}\left(\omega^{i}\right)$, defined inside the star $\omega^{i}$, verify the local equation

$$
\int_{\omega^{i}}\left[\nu \hat{\boldsymbol{q}}^{i} \cdot \boldsymbol{\nabla} v+\tilde{\sigma} \hat{r}^{i} v\right] \mathrm{d} \Omega+\frac{1}{2} \int_{\Gamma_{\mathrm{N}} \cap \partial \omega^{i}} \boldsymbol{\alpha} \cdot \boldsymbol{n} \hat{r}^{i} v \mathrm{~d} \Gamma=R^{*}\left(\phi^{i} v\right) \quad \forall v \in \mathcal{V}\left(\omega^{i}\right) .
$$

REMARK 5. It is tacitly assumed that the problems given in equation (5.3) have at least one solution. A strictly positive reaction term in the l.h.s., $\tilde{\sigma}>0$, ensures the solvability of local equation (5.3). For $\left.\tilde{\sigma}\right|_{\omega^{i}}=0$, the kernel of the bilinear operator appearing in the l.h.s. is the one dimensional space of constants, $\mathbb{P}^{0}\left(\omega^{i}\right)$. Then, equation (5.3) is solvable if and only if the compatibility condition holds, namely

$$
R^{*}\left(\phi^{i} c\right)=c R^{*}\left(\phi^{i}\right)=0 \quad \forall c \in \mathbb{P}^{0}\left(\omega^{i}\right),
$$

which follows from the orthogonality of the primal and adjoint residuals to the finite element space $\mathcal{V}^{h}$ (i.e. $\left.R^{*}(v)=0 \forall v \in \mathcal{V}^{h}\right)$, since $\phi^{i} \in \mathcal{V}^{h}$.

THEOREM 5.1. The dual estimates $\hat{\boldsymbol{q}}=\sum_{i=1}^{n_{\mathrm{np}}} \hat{\boldsymbol{q}}^{i}$ and $\hat{r}=\sum_{i=1}^{n_{\mathrm{np}}} \hat{r}^{i}$, where $\hat{\boldsymbol{q}}^{i}$ and $\hat{r}^{i}$ are the solutions of the local problem (5.3), fulfill the hypothesis of theorem 4.1 and therefore

$$
\|z\|^{2} \leq \int_{\Omega}\left[\nu \hat{\boldsymbol{q}} \cdot \hat{\boldsymbol{q}}+\tilde{\sigma} \hat{r}^{2}\right] \mathrm{d} \Omega+\frac{1}{2} \int_{\Gamma_{\mathrm{N}}} \boldsymbol{\alpha} \cdot \boldsymbol{n} \hat{r}^{2} \mathrm{~d} \Gamma .
$$

Proof. The dual estimates $\hat{\boldsymbol{q}}$ and $\hat{r}$ verify equation (4.2) and therefore theorem 5.1 is a straightforward particularization of theorem 4.1. Indeed, let $v \in \mathcal{V}$, then using the definition of the dual estimates, equation (5.2), and the local equations (5.3) - note that if $v \in \mathcal{V}$ then $\left.v\right|_{\omega^{i}} \in \mathcal{V}\left(\omega^{i}\right)$ - then,

$$
\begin{aligned}
\int_{\Omega}[\nu \hat{\boldsymbol{q}} \cdot & \nabla v+\tilde{\sigma} \hat{r} v] \mathrm{d} \Omega+\frac{1}{2} \int_{\Gamma_{\mathrm{N}}} \boldsymbol{\alpha} \cdot \boldsymbol{n} \hat{r} v \mathrm{~d} \Gamma \\
= & \sum_{i=1}^{n_{\mathrm{np}}}\left\{\int_{\omega^{i}}\left[\nu \hat{\boldsymbol{q}}^{i} \cdot \boldsymbol{\nabla} v+\tilde{\sigma} \hat{r}^{i} v\right] \mathrm{d} \Omega+\frac{1}{2} \int_{\Gamma_{\mathrm{N}} \cap \partial \omega^{i}} \boldsymbol{\alpha} \cdot \boldsymbol{n} \hat{r}^{i} v \mathrm{~d} \Gamma\right\} \\
= & \sum_{i=1}^{n_{\mathrm{np}}} R^{*}\left(\phi^{i} v\right)=R^{*}\left(\sum_{i=1}^{n_{\mathrm{np}}} \phi^{i} v\right)=R^{*}(v)=a^{\mathrm{s}}(z, v)
\end{aligned}
$$

where in the final equalities the linearity of the residual $R^{*}(\cdot)$, the partition-of-unity property, equation (5.1), and the definition of $z$, equation (4.1), have also been used.

5.2. Strong form of the local problems for the dual estimates $\hat{\boldsymbol{q}}^{i}$ and $\hat{r}^{i}$. This section is intended to provide a sketch of the computation of the piecewise polynomial dual estimates $\hat{\boldsymbol{q}}^{i} \in\left[\widehat{\mathbb{P}}^{q}\left(\omega^{i}\right)\right]^{2}$ and $\hat{r}^{i} \in \widehat{\mathbb{P}}^{q}\left(\omega^{i}\right)$ solution of (5.3). Actually, for ease of explanation, this section provides the strong form of the local error equation (5.3). A more detailed construction of the dual estimates may be found in [25].

The r.h.s. of equation (5.3), $R^{*}\left(\phi_{i} v\right)$, is rewritten as

$$
R^{*}\left(\phi_{i} v\right)=\int_{\omega^{i}} f_{i}^{*} v \mathrm{~d} \Omega+\int_{\Gamma_{N} \cap \partial \omega^{i}} g_{i}^{*} v \mathrm{~d} \Gamma-\int_{\omega^{i}} \nu \hat{\boldsymbol{q}}_{h}^{i} \cdot \nabla v \mathrm{~d} \Omega
$$


where the following compact notation is introduced

$$
\begin{gathered}
f_{i}^{*}=\alpha\left[\phi_{i} f-\phi_{i} \boldsymbol{\alpha} \cdot \nabla u_{h}-\sigma \phi_{i} u_{h}-\nu \boldsymbol{\nabla} u_{h} \cdot \boldsymbol{\nabla} \phi_{i}\right]+\beta\left[\phi_{i} f^{\mathcal{O}}-\psi_{h} \boldsymbol{\alpha} \cdot \boldsymbol{\nabla} \phi_{i}-\sigma \phi_{i} \psi_{h}-\nu \boldsymbol{\nabla} \psi_{h} \cdot \boldsymbol{\nabla} \phi_{i}\right], \\
g_{i}^{*}=\alpha \phi_{i} g+\beta \phi_{i} g^{\mathcal{O}} \quad \text { and } \quad \hat{\boldsymbol{q}}_{h}^{i}=\alpha \phi_{i} \boldsymbol{\nabla} u_{h}+\beta\left(\phi_{i} \boldsymbol{\nabla} \psi_{h}+\frac{1}{\nu} \phi_{i} \psi_{h} \boldsymbol{\alpha}\right) .
\end{gathered}
$$

Thus, equation (5.3) is modified into

$$
\int_{\omega^{i}}\left[\nu \hat{\boldsymbol{q}}_{*}^{i} \cdot \boldsymbol{\nabla} v+\tilde{\sigma} \hat{r}^{i} v\right] \mathrm{d} \Omega+\frac{1}{2} \int_{\Gamma_{\mathrm{N}} \cap \partial \omega^{i}} \boldsymbol{\alpha} \cdot \boldsymbol{n} \hat{r}^{i} v \mathrm{~d} \Gamma=\int_{\omega^{i}} f_{i}^{*} v \mathrm{~d} \Omega+\int_{\Gamma_{N} \cap \partial \omega^{i}} g_{i}^{*} v \mathrm{~d} \Gamma
$$

introducing the new unknown $\hat{\boldsymbol{q}}_{*}^{i}=\hat{\boldsymbol{q}}^{i}+\hat{\boldsymbol{q}}_{h}^{i}$.

The 1.h.s. of equation (5.5) is rewritten, applying the divergence theorem, as

$$
\int_{\omega^{i}}\left[-\nu \nabla \cdot \hat{\boldsymbol{q}}_{*}^{i}+\tilde{\sigma} \hat{r}^{i}\right] v \mathrm{~d} \Omega+\frac{1}{2} \int_{\Gamma_{\mathrm{N}} \cap \partial \omega^{i}} \boldsymbol{\alpha} \cdot \boldsymbol{n} \hat{r}^{i} v \mathrm{~d} \Gamma+\sum_{\Omega_{k} \subset \omega^{i}} \int_{\partial \Omega_{k}} \nu \hat{\boldsymbol{q}}_{*}^{i} \cdot \boldsymbol{n} v \mathrm{~d} \Gamma .
$$

Therefore, substituting (5.5) into (5.6), an alternative form of equation (5.3) is: find $\hat{\boldsymbol{q}}_{*}^{i} \in\left[\widehat{\mathbb{P}}^{q}\left(\omega^{i}\right)\right]^{2}$ and $\hat{r}^{i} \in \widehat{\mathbb{P}}^{q}\left(\omega^{i}\right)$ such that

$$
\begin{gathered}
\int_{\omega^{i}}\left[-\nu \nabla \cdot \hat{\boldsymbol{q}}_{*}^{i}+\tilde{\sigma} \hat{r}^{i}\right] v \mathrm{~d} \Omega+\frac{1}{2} \int_{\Gamma_{\mathrm{N}} \cap \partial \omega^{i}} \boldsymbol{\alpha} \cdot \boldsymbol{n} \hat{r}^{i} v \mathrm{~d} \Gamma+\sum_{\Omega_{k} \subset \omega^{i}} \int_{\partial \Omega_{k}} \nu \hat{\boldsymbol{q}}_{*}^{i} \cdot \boldsymbol{n} v \mathrm{~d} \Gamma \\
=\int_{\omega^{i}} f_{i}^{*} v \mathrm{~d} \Omega+\int_{\Gamma_{N} \cap \partial \omega^{i}} g_{i}^{*} v \mathrm{~d} \Gamma \quad \text { for all } v \in \mathcal{V}\left(\omega^{i}\right)
\end{gathered}
$$

and recover $\hat{\boldsymbol{q}}^{i}=\hat{\boldsymbol{q}}_{*}^{i}-\hat{\boldsymbol{q}}_{h}^{i}$.

The strong form to compute the dual estimates $\hat{\boldsymbol{q}}_{*}^{i} \in\left[\widehat{\mathbb{P}}^{q}\left(\omega^{i}\right)\right]^{2}$ and $\hat{r}^{i} \in \widehat{\mathbb{P}}^{q}\left(\omega^{i}\right)$ follows identifying terms in (5.7),

$$
\begin{array}{cc}
-\nu \nabla \cdot \hat{\boldsymbol{q}}_{*}^{i}+\tilde{\sigma} \hat{r}^{i}=f_{i}^{*} & \text { in } \omega^{i} \\
\nu \hat{\boldsymbol{q}}_{*}^{i} \cdot \boldsymbol{n}+\frac{1}{2} \boldsymbol{\alpha} \cdot \boldsymbol{n} \hat{r}^{i}=g_{i}^{*} & \text { on } \gamma \in \Gamma_{\mathrm{N}} \cap \partial \omega^{i} \\
\nu \hat{\boldsymbol{q}}_{*}^{i} \cdot \boldsymbol{n}=0 & \text { on } \gamma \in \partial \omega^{i}-\left\{\Gamma_{\mathrm{N}} \cup \Gamma_{\mathrm{D}}\right\} \\
\left.\nu \hat{\boldsymbol{q}}_{*}^{i}\right|_{\Omega_{k}} \cdot \boldsymbol{n}_{k}+\left.\nu \hat{\boldsymbol{q}}_{*}^{i}\right|_{\Omega_{l}} \cdot \boldsymbol{n}_{l}=0 & \text { on } \gamma \in \partial \Omega_{k} \cap \partial \Omega_{l}, \quad \Omega_{k}, \Omega_{l} \subset \omega^{i},
\end{array}
$$

where $\boldsymbol{n}_{k}$ and $\boldsymbol{n}_{l}$ are the outward normal to the elements $\Omega_{k}$ and $\Omega_{l}$ respectively.

REMARK 6. Any collection of pairs $\hat{\boldsymbol{q}}_{*}^{i}, \hat{r}^{i} i=1, \cdots, n_{\mathrm{np}}$ fulfilling these equations provides global estimates $\hat{\boldsymbol{q}}$ and $\hat{r}$ yielding upper bounds of $\|z\|$. Nevertheless, the previous equations do not uniquely determine the dual estimates $\hat{\boldsymbol{q}}_{*}^{i} \in\left[\widehat{\mathbb{P}}^{q}\left(\omega^{i}\right)\right]^{2}$ and $\hat{r}^{i} \in \widehat{\mathbb{P}}^{q}\left(\omega^{i}\right)$. The additional degrees of freedom may be used to minimize the final bounds.

REMARK 7. Given any set of couple $\hat{\boldsymbol{q}}_{*}^{i}, \hat{r}^{i} i=1, \cdots, n_{\mathrm{np}}$, the upper bound for $\|z\|^{2}$ is

$$
\begin{aligned}
\int_{\Omega}[\nu \hat{\boldsymbol{q}} \cdot \hat{\boldsymbol{q}} & \left.+\tilde{\sigma} \hat{r}^{2}\right] \mathrm{d} \Omega+\frac{1}{2} \int_{\Gamma_{\mathrm{N}}} \boldsymbol{\alpha} \cdot \boldsymbol{n} \hat{r}^{2} \mathrm{~d} \Gamma \\
& =\int_{\Omega}\left[\nu\left(\sum_{i=1}^{n_{\mathrm{np}}} \hat{\boldsymbol{q}}^{i}\right) \cdot\left(\sum_{i=1}^{n_{\mathrm{np}}} \hat{\boldsymbol{q}}^{i}\right)+\tilde{\sigma}\left(\sum_{i=1}^{n_{\mathrm{np}}} \hat{r}^{i}\right)^{2}\right] \mathrm{d} \Omega+\frac{1}{2} \int_{\Gamma_{\mathrm{N}}} \boldsymbol{\alpha} \cdot \boldsymbol{n}\left(\sum_{i=1}^{n_{\mathrm{np}}} \hat{r}^{i}\right)^{2} \mathrm{~d} \Gamma,
\end{aligned}
$$


thus, the determination of the estimates $\hat{\boldsymbol{q}}_{*}^{i}, \hat{r}^{i}$ minimizing the upper bound requires coupling all the local problems. Here, the simplest and straightforward criteria to select one of the multiple pairs $\hat{\boldsymbol{q}}_{*}^{i}, \hat{r}^{i}$ is chosen: the local estimates $\hat{\boldsymbol{q}}_{*}^{i}, \hat{r}^{i}$ are required to minimize the local norm

$$
\int_{\omega^{i}}\left[\nu \hat{\boldsymbol{q}}^{i} \cdot \hat{\boldsymbol{q}}^{i}+\tilde{\sigma}\left(\hat{r}^{i}\right)^{2}\right] \mathrm{d} \Omega+\frac{1}{2} \int_{\Gamma_{N} \cap \partial \omega^{i}} \boldsymbol{\alpha} \cdot \boldsymbol{n}\left(\hat{r}^{i}\right)^{2} \mathrm{~d} \Gamma .
$$

Other alternative choices may be found in [25].

6. Bounds for the quantity of interest $s=\ell^{\mathcal{O}}(u)$ : an algorithmic summary. According to theorem 3.1, upper and lower bounds of $s=\ell^{\mathcal{O}}(u)$ are available once upper bounds of the energy norm $\|z\|$ are obtained for the two combinations $(\alpha, \beta)=(\kappa, 1 / \kappa)$ and $(\alpha, \beta)=(\kappa,-1 / \kappa)$. The general strategy to obtain these upper bounds is described in the previous section. Due to the linearity of the problem, obtaining the estimates for the two values $z=\kappa e^{\mathrm{s}} \pm 1 / \kappa \varepsilon^{\mathrm{s}}$ is equivalent to obtain the estimates for $z=e^{\mathrm{s}}$ and $z=\varepsilon^{\mathrm{s}}$, that is for the two combinations $(\alpha, \beta)=(1,0)$ and $(\alpha, \beta)=(0,1)$.

The main steps of the procedure to compute bounds for $\ell^{\mathcal{O}}(u)$ are the following:

1. Compute the primal and adjoint solutions $u_{h}$ and $\psi_{h}$ respectively.

2. For each star $\omega^{i}$ (associated with the node $\boldsymbol{x}^{i}$ of the mesh) compute the primal and adjoint dual estimates $\hat{\boldsymbol{q}}_{P}^{i}, \hat{\boldsymbol{q}}_{D}^{i} \in\left[\widehat{\mathbb{P}}^{q}\left(\omega^{i}\right)\right]^{2}$ and $\hat{r}_{P}^{i}, \hat{r}_{D}^{i} \in \widehat{\mathbb{P}}^{q}\left(\omega^{i}\right)$ such that

$\int_{\omega^{i}}\left[\nu \hat{\boldsymbol{q}}_{P}^{i} \cdot \nabla v+\tilde{\sigma} \hat{r}_{P}^{i} v\right] \mathrm{d} \Omega+\frac{1}{2} \int_{\Gamma_{\mathrm{N}} \cap \partial \omega^{i}} \boldsymbol{\alpha} \cdot \boldsymbol{n} \hat{r}_{P}^{i} v \mathrm{~d} \Gamma=R^{\mathrm{P}}\left(\phi^{i} v\right) \quad \forall v \in \mathcal{V}\left(\omega^{i}\right)$,

and

$\int_{\omega^{i}}\left[\nu \hat{\boldsymbol{q}}_{D}^{i} \cdot \nabla v+\tilde{\sigma} \hat{r}_{D}^{i} v\right] \mathrm{d} \Omega+\frac{1}{2} \int_{\Gamma_{\mathrm{N}} \cap \partial \omega^{i}} \boldsymbol{\alpha} \cdot \boldsymbol{n} \hat{r}_{D}^{i} v \mathrm{~d} \Gamma=R^{\mathrm{D}}\left(\phi^{i} v\right) \quad \forall v \in \mathcal{V}\left(\omega^{i}\right)$,

minimizing

$$
\int_{\omega^{i}}\left[\nu \hat{\boldsymbol{q}}_{P}^{i} \cdot \hat{\boldsymbol{q}}_{P}^{i}+\tilde{\sigma}\left(\hat{r}_{P}^{i}\right)^{2}\right] \mathrm{d} \Omega+\frac{1}{2} \int_{\Gamma_{N} \cap \partial \omega^{i}} \boldsymbol{\alpha} \cdot \boldsymbol{n}\left(\hat{r}_{P}^{i}\right)^{2} \mathrm{~d} \Gamma
$$

and

$$
\int_{\omega^{i}}\left[\nu \hat{\boldsymbol{q}}_{D}^{i} \cdot \hat{\boldsymbol{q}}_{D}^{i}+\tilde{\sigma}\left(\hat{r}_{D}^{i}\right)^{2}\right] \mathrm{d} \Omega+\frac{1}{2} \int_{\Gamma_{N} \cap \partial \omega^{i}} \boldsymbol{\alpha} \cdot \boldsymbol{n}\left(\hat{r}_{D}^{i}\right)^{2} \mathrm{~d} \Gamma
$$

respectively.

3 Recover the global estimates

$$
\hat{\boldsymbol{q}}_{P}=\sum_{i=1}^{n_{\mathrm{np}}} \hat{\boldsymbol{q}}_{P}^{i}, \quad \hat{r}_{P}=\sum_{i=1}^{n_{\mathrm{np}}} \hat{r}_{P}^{i} \quad \text { and } \quad \hat{\boldsymbol{q}}_{D}=\sum_{i=1}^{n_{\mathrm{np}}} \hat{\boldsymbol{q}}_{D}^{i}, \quad \hat{r}_{D}=\sum_{i=1}^{n_{\mathrm{np}}} \hat{r}_{D}^{i} .
$$

4 Compute the three scalar quantities

$$
\begin{aligned}
\left(\eta^{P}\right)^{2} & :=\sum_{k=1}^{n_{\mathrm{el}}} \eta_{k}^{P}=\sum_{k=1}^{n_{\mathrm{el}}} \int_{\Omega_{k}}\left[\nu \hat{\boldsymbol{q}}_{P} \cdot \hat{\boldsymbol{q}}_{P}+\tilde{\sigma}\left(\hat{r}_{P}\right)^{2}\right] \mathrm{d} \Omega+\frac{1}{2} \int_{\Gamma_{\mathrm{N}} \cap \partial \Omega_{k}} \boldsymbol{\alpha} \cdot \boldsymbol{n}\left(\hat{r}_{P}\right)^{2} \mathrm{~d} \Gamma, \\
\left(\eta^{D}\right)^{2} & :=\sum_{k=1}^{n_{\mathrm{el}}} \eta_{k}^{D}=\sum_{k=1}^{n_{\mathrm{el}}} \int_{\Omega_{k}}\left[\nu \hat{\boldsymbol{q}}_{D} \cdot \hat{\boldsymbol{q}}_{D}+\tilde{\sigma}\left(\hat{r}_{D}\right)^{2}\right] \mathrm{d} \Omega+\frac{1}{2} \int_{\Gamma_{\mathrm{N}} \cap \partial \Omega_{k}} \boldsymbol{\alpha} \cdot \boldsymbol{n}\left(\hat{r}_{D}\right)^{2} \mathrm{~d} \Gamma,
\end{aligned}
$$




$$
\eta^{P D}:=\sum_{k=1}^{n_{\mathrm{el}}} \eta_{k}^{P D}=\sum_{k=1}^{n_{\mathrm{el}}} \int_{\Omega_{k}}\left[\nu \hat{\boldsymbol{q}}_{P} \cdot \hat{\boldsymbol{q}}_{D}+\tilde{\sigma} \hat{r}_{P} \hat{r}_{D}\right] \mathrm{d} \Omega+\frac{1}{2} \int_{\Gamma_{\mathrm{N}} \cap \partial \Omega_{k}} \boldsymbol{\alpha} \cdot \boldsymbol{n} \hat{r}_{P} \hat{r}_{D} \mathrm{~d} \Gamma .
$$

5. Recover the bounds for the output $s^{l b} \leq s \leq s^{u b}$ as

$$
s^{l b}:=s_{h}-\frac{1}{2} \eta^{P} \eta^{D}+\frac{1}{2} \eta^{P D} \leq s \leq s_{h}+\frac{1}{2} \eta^{P} \eta^{D}+\frac{1}{2} \eta^{P D}=: s^{u b},
$$

where $s_{h}=\ell^{\mathcal{O}}\left(u_{h}\right)$.

REMARK 8. The final expression for the bounds of the output $\ell^{\mathcal{O}}(u)$, see equation (6.1), is derived by the following rationale. First theorem 3.1 states that in order to obtain bounds for the error in the output $s$ it is sufficient to obtain upper bounds for the quantities $\| \kappa e^{\mathrm{s}} \pm$ $\frac{1}{\kappa} \varepsilon^{\mathrm{s}} \|_{u b}^{2}$, see equation (3.6). In order to compute these upper bounds, the procedure detailed in section 4 is considered for $z=\kappa e^{\mathrm{s}} \pm \frac{1}{\kappa} \varepsilon^{\mathrm{s}}$. Then, from theorem 5.1, the following upper bounds are obtained:

$$
\begin{aligned}
\left\|\kappa e^{\mathrm{s}} \pm \frac{1}{\kappa} \varepsilon^{\mathrm{s}}\right\|_{u b}^{2}= & \int_{\Omega}\left[\nu\left(\kappa \hat{\boldsymbol{q}}_{P} \pm \frac{1}{\kappa} \hat{\boldsymbol{q}}_{D}\right) \cdot\left(\kappa \hat{\boldsymbol{q}}_{P} \pm \frac{1}{\kappa} \hat{\boldsymbol{q}}_{D}\right)+\tilde{\sigma}\left(\kappa \hat{r}_{P} \pm \frac{1}{\kappa} \hat{r}_{D}\right)^{2}\right] \mathrm{d} \Omega \\
& +\frac{1}{2} \int_{\Gamma_{\mathrm{N}}} \boldsymbol{\alpha} \cdot \boldsymbol{n}\left(\kappa \hat{r}_{P} \pm \frac{1}{\kappa} \hat{r}_{D}\right)^{2} \mathrm{~d} \Gamma .
\end{aligned}
$$

Finally the expressions for the bounds are obtained expanding the previous expression, taking $\kappa^{2}=\eta^{D} / \eta^{P}$ and rearranging terms.

REMARK 9. The local dual problems to determine $\hat{\boldsymbol{q}}_{P}^{i}, \hat{\boldsymbol{q}}_{D}^{i} \in\left[\widehat{\mathbb{P}}^{q}\left(\omega^{i}\right)\right]^{2}$ and $\hat{r}_{P}^{i}, \hat{r}_{D}^{i} \in$ $\widehat{\mathbb{P}}^{q}\left(\omega^{i}\right)$ can be solved in the same manner as the local problems given in equation (5.3) - see for instance section (5.2) where the strong form of the local problems is given. Also the details on the implementation of the local problems may be found in [25]. The important point is that once the finite element approximations $u_{h}$ and $\psi_{h}$ have been computed, the exact bounds for the output can be computed with asymptotically linear cost in the number of vertices of the finite element discretization. Moreover the local dual subproblems computation may be parallelized.

7. Numerical examples. In the following, the bound average $s^{a v e}:=\left(s^{u b}+s^{l b}\right) / 2$ is taken as a new approximation of the quantity of interest and the half bound gap $\Delta=\left(s^{u b}\right.$ $\left.s^{l b}\right) / 2$ is seen as an error indicator. The relative counterpart of the bound gap $\Delta_{\text {rel }}=\Delta / s^{a v e}$ is also used in the presentation.

The meshes are adapted to reduce the half bound gap $\Delta$. In the examples a simple adaptive strategy is used based on the decomposition of $\Delta$ into local positive contributions from the elements:

$$
\Delta=\sum_{k=1}^{n_{\mathrm{el}}} \Delta_{k}
$$

where the element contribution to the bound gap $\Delta_{k}$ is

$$
\Delta_{k}:=\frac{1}{4} \kappa^{2} \eta_{k}^{P}+\frac{1}{4 \kappa^{2}} \eta_{k}^{D} .
$$

Note that this decomposition is valid because

$$
\Delta=\frac{s^{u b}-s^{l b}}{2}=\frac{1}{2} \eta^{P} \eta^{D}=\frac{1}{4} \kappa^{2}\left(\eta^{P}\right)^{2}+\frac{1}{4 \kappa^{2}}\left(\eta^{D}\right)^{2}=\sum_{k=1}^{n_{\mathrm{el}}}\left[\frac{1}{4} \kappa^{2} \eta_{k}^{P}+\frac{1}{4 \kappa^{2}} \eta_{k}^{D}\right]=\sum_{k=1}^{n_{\mathrm{el}}} \Delta_{k} .
$$


The remeshing strategy consists in subdividing the elements with the larger values of $\Delta_{k}$ at each step of the adaptive procedure.

The behavior of the bounds introduced in the present work is compared with the three different strategies presented in [22, 19, 30]. The strategy presented in [22] also solves local problems in subdomains (therefore avoiding the computation of equilibrated fluxes), but the local problems are solved using a local fine submesh. This yields bounds which are only guaranteed in the asymptotic regime. The strategy presented in [19] is a classical hybrid flux method where first the (linear) equilibrated fluxes are computed and then the local elementary problems are solved using a local fine submesh. Finally the results are compared to the strategy presented in [30] which also provides strict bounds for the output and differs from [19] in the solution of the local elementary problems. Instead of using a local submesh, dual estimates are computed to recover strict bounds for the output.

The bounds computed using the strategies presented in [22], [19] and [30] will be denoted in the following as asymptotic flux-free bounds, asymptotic equilibrated bounds and strict equilibrated bounds respectively.

7.1. Example 1: uniformly forced square domain. The pure diffusion equation $(\nu=$ $1, \sigma=0, \boldsymbol{\alpha}=\mathbf{0}$ in (2.1)) is solved in the squared domain $\Omega=[0,1] \times[0,1]$. A constant source term $f=\sqrt{10}$ and homogeneous Dirichlet boundary conditions are considered.

The quantity of interest is an average of the solution,

$$
\ell^{\mathcal{O}}(u)=\int_{\Omega} \sqrt{10} u(x, y) \mathrm{d} \Omega,
$$

that is $f^{\mathcal{O}}=\sqrt{10}$ and $g^{\mathcal{O}}=0$ in equation (2.2). In this case, the solution $\psi$ of the adjoint problem coincides with the primal solution, $\psi=u$. It is well known that in this case, the finite element approximation of the output is a lower bound for $s, \ell^{\mathcal{O}}\left(u_{h}\right) \leq s$. The present methodology, as well as the strategies presented in [22, 30, 19], yields $s^{l b}=\ell^{\mathcal{O}}\left(u_{h}\right)$.

Linear triangular elements, $p=1$, are used for the computation of the primal and adjoint finite element approximations, and the local dual approximations $\hat{\boldsymbol{q}}^{i} \in\left[\widehat{\mathbb{P}}^{3}\left(\omega^{i}\right)\right]^{2}$ and $\hat{r}^{i} \in$ $\widehat{\mathbb{P}}^{3}\left(\omega^{i}\right)$ are piecewise third order polynomials, i.e. $q=3$.

The convergence of the bounds is analyzed for a uniform mesh refinement in a series of structured meshes. The initial mesh is composed of 8 triangular elements (half squares) and in each refinement step every triangle is divided into four similar triangles. The results are displayed in tables 7.1 and in figure 7.1. Since for this particular case all the methodologies yield $s^{l b}=s_{h}=\ell^{\mathcal{O}}\left(u_{h}\right)$, only the upper bounds are compared. For this problem the exact solution $s=0.3514425$ is known, and the effectiveness of the bounds are computed as

$$
\theta^{*}=\frac{\left|s-s^{*}\right|}{|s|},
$$

where the symbol $*$ stands for $u b, l b$ or ave. Note that $\theta$ measures the quality of the estimator: the lower values of $\theta$ correspond to the sharper estimates. This effectivity index is only available for this example and the next one (examples 1 and 2) because in the following the exact solution is not known.

As expected, the upper bounds provided by the asymptotic strategies are lower, and hence sharper, than the corresponding upper bounds obtained using strict strategies. However, this is not always a positive feature: for the first three meshes (the coarsest) and for the mesh of 2048 elements the asymptotic upper bound is not an actual upper bound of the exact solution. Since the asymptotic upper bound estimates are only guaranteed to overestimate the reference values, for some meshes (not necessarily the coarsest ones) they do underestimate the exact value $s$. 


\begin{tabular}{|c|cc||cc|cc|}
\hline \multicolumn{2}{|l||}{} & \multicolumn{2}{c||}{} & \multicolumn{2}{c|}{ asymp. flux-free } \\
\hline$n_{\mathrm{el}}$ & $s_{h}$ & $\theta^{h}$ & $s^{u b}$ & $\theta^{u b}$ & $s^{u b}$ & $\theta^{u b}$ \\
\hline 8 & 0.156250 & $55.54 \%$ & 0.358184 & $1.92 \%$ & 0.346651 & $1.36 \%$ \\
32 & 0.288086 & $18.03 \%$ & 0.352852 & $0.40 \%$ & 0.349984 & $0.42 \%$ \\
128 & 0.334230 & $4.90 \%$ & 0.352071 & $0.18 \%$ & 0.351404 & $0.01 \%$ \\
512 & 0.347028 & $1.26 \%$ & 0.351646 & $0.06 \%$ & 0.351491 & $0.01 \%$ \\
2048 & 0.350330 & $0.32 \%$ & 0.351500 & $0.02 \%$ & 0.351282 & $0.05 \%$ \\
8192 & 0.351164 & $0.08 \%$ & 0.351458 & $0.004 \%$ & - & - \\
\hline \hline & & & \multicolumn{2}{|c||}{ strict equilibrated } & asymp. equilibrated \\
\hline$n_{\mathrm{el}}$ & & & $s^{u b}$ & $\theta^{u b}$ & $s^{u b}$ & $\theta^{u b}$ \\
\hline 8 & & & 0.630232 & $79.33 \%$ & 0.577370 & $64.29 \%$ \\
32 & & & 0.445664 & $26.81 \%$ & 0.429033 & $22.08 \%$ \\
128 & & & 0.377467 & $7.40 \%$ & 0.372986 & $6.13 \%$ \\
512 & & & 0.358125 & $1.90 \%$ & 0.356984 & $1.58 \%$ \\
2048 & & & 0.353122 & $0.48 \%$ & 0.351796 & $0.10 \%$ \\
8192 & & & 0.351862 & $0.12 \%$ & - & - \\
\hline
\end{tabular}

TABLE 7.1

Example 1: series of uniformly h-refined linear triangular meshes.
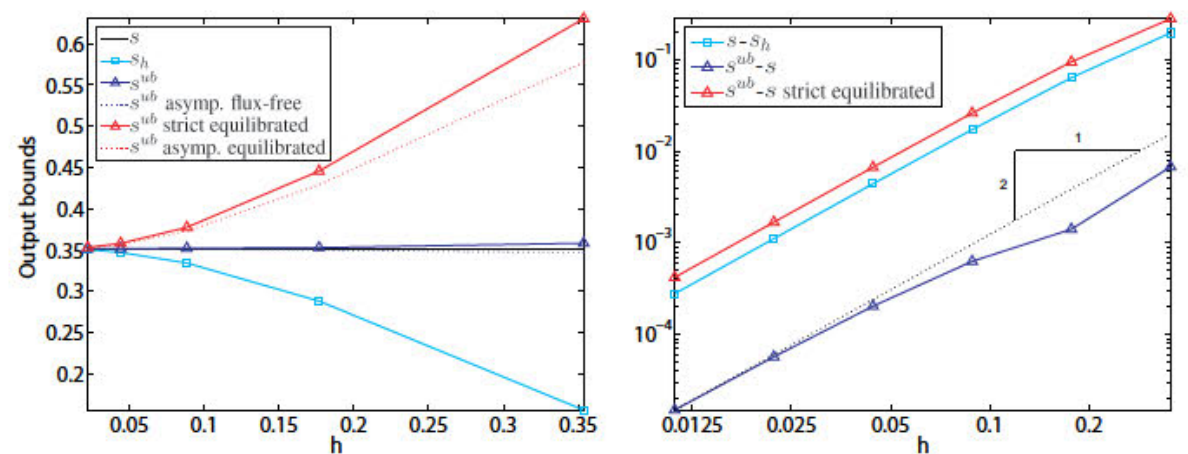

FIG. 7.1. Example 1: series of uniformly h-refined linear triangular meshes. Bounds (left) and their convergence (right).

The evolution of the strict bounds (both for the equilibrated and for the flux-free strategies) shows the optimal finite element asymptotic convergence rate of $\mathcal{O}\left(h^{2}\right)$, see figure 7.1. Moreover, the bounds obtained by the flux-free strategy provide much better effectivities, that is much lower and sharper upper bounds. Note that using flux-free instead of the equilibrated fluxes approach, the effectivity index $\theta$ is reduced in approximately two orders of magnitude. Also in this example the upper bounds provided by the flux-free strategy provide better effectivities than the finite element approximations (the index $\theta$ is reduced in approximately one order of magnitude).

7.2. Example 2: quasi-2D transport. This example is the quasi-2D transport problem introduced in [30] with known analytical solution. The effect of including the convective term is analyzed in this simple problem for different values of the velocity $\alpha$. Equation (2.1) is solved in the unit square $\Omega=[0,1] \times[0,1]$, for $\nu=1$ and a uniform horizontal velocity field $\alpha=(\alpha, 0)$. The performance of the introduced estimates is tested for different values 
of $\alpha$. The boundary conditions are of Dirichlet type on the lateral sides, homogeneous on the right $u(1, y)=0$ and set to 1 on the left $u(0, y)=1$. The boundary condition on both the top and bottom are Neumann homogeneous and the source term is $f=0$. The degrees of the interpolation spaces are $p=1$ and $q=3$.

The quantity of interest is an overall average of the solution, that is

$$
\ell^{\mathcal{O}}(u)=\int_{\Omega} u(x, y) \mathrm{d} \Omega
$$

which corresponds to $f^{\mathcal{O}}=1$.

The error estimation strategies and the computation of bounds are performed for a series of uniformly $h$-refined meshes for $\sigma=1$ and different values of $\alpha$. The results are displayed in table 7.2 and figure 7.2. For all the values of $\alpha$, the rate of convergence of the bound gap is found to be equal to the expected one for the error, that is $\mathcal{O}\left(h^{2}\right)$. It is worth noting that the bound gap is larger as $\alpha$ increases. For $\alpha=100$ the bound gap is 4 orders of magnitude larger than for $\alpha=0$, being the quantity of interest of the same order.

\begin{tabular}{c|cc|cc|cc|cc}
\hline & \multicolumn{2}{|c|}{$\alpha=0$} & \multicolumn{2}{c|}{$\alpha=1$} & \multicolumn{2}{c|}{$\alpha=5$} & \multicolumn{2}{c}{$\alpha=10$} \\
& \multicolumn{2}{|c|}{$s=0.462117$} & \multicolumn{2}{c|}{$s=0.536142$} & \multicolumn{2}{c}{$s=0.755101$} & \multicolumn{2}{c}{$s=0.862436$} \\
\hline$n_{\mathrm{el}}$ & $s^{\text {ave }}$ & $\Delta$ & $s^{\text {ave }}$ & $\Delta$ & $s^{\text {ave }}$ & $\Delta$ & $s^{\text {ave }}$ & $\Delta$ \\
\hline \hline 8 & 0.466353 & 0.005475 & 0.532597 & 0.006295 & 0.73043 & 0.1079 & 0.66223 & 0.5395 \\
32 & 0.463163 & 0.001384 & 0.535319 & 0.001635 & 0.75306 & 0.0285 & 0.86218 & 0.1239 \\
128 & 0.462380 & 0.000345 & 0.535939 & 0.000411 & 0.75465 & 0.0078 & 0.86236 & 0.0312 \\
512 & 0.462183 & 0.000086 & 0.536092 & 0.000103 & 0.75499 & 0.0018 & 0.86242 & 0.0077 \\
2048 & 0.462134 & 0.000022 & 0.536130 & 0.000026 & 0.75507 & 0.0005 & 0.86243 & 0.0019 \\
8192 & 0.462121 & 0.000005 & 0.536139 & 0.000006 & 0.75509 & 0.0001 & 0.86244 & 0.0005 \\
\hline \hline 8 & 0.462675 & 0.009420 & 0.535946 & 0.010560 & 0.74147 & 0.1650 & 0.58587 & 0.7827 \\
32 & 0.462317 & 0.002347 & 0.536002 & 0.002749 & 0.75425 & 0.0462 & 0.86165 & 0.1938 \\
128 & 0.462173 & 0.000586 & 0.536099 & 0.000696 & 0.75491 & 0.0121 & 0.86218 & 0.0514 \\
512 & 0.462132 & 0.000146 & 0.536131 & 0.000174 & 0.75505 & 0.0031 & 0.86237 & 0.0131 \\
2048 & 0.462121 & 0.000037 & 0.536139 & 0.000044 & 0.75509 & 0.0008 & 0.86242 & 0.0033 \\
8192 & 0.462118 & 0.000009 & 0.536142 & 0.000011 & 0.75510 & 0.0002 & 0.86243 & 0.0008 \\
\hline
\end{tabular}

TABLE 7.2

Example 2: results in a series of uniformly h-refined meshes for $\sigma=1$ : flux-free (top) and hybrid-flux (bottom).

This increment in the bound gap does not correspond to the actual error increment and therefore it has to be concluded that the efficiency of the computed error bounds deteriorate if the advection parameter is large. This very same phenomenon is observed with asymptotic estimates. The fact of obtaining the exact bounds is not introducing any further degradation of significant improvement in the influence of the advection parameter in the results.

Tables 7.3 and 7.4 summarize the influence of the Peclet number $\alpha$ on the effectivity of the bounds in the context of the simple adaptive method with a tolerance of $\Delta_{t o l}=0.001 \mathrm{~s}$ and for $\sigma=10$. Although the method is valid for nonnegative $\alpha$, the sharpness of the bound degrades significantly with increasing Peclet number, but the bounding property is retained. Since we know the exact output for this example, we can calculate the effectiveness of the bounds as an indicator of the error in the finite element solution using

$$
\eta=\frac{s^{u b}-s^{l b}}{2\left|s-s_{h}\right|}=\frac{\Delta}{\left|s-s_{h}\right|}=\frac{\theta^{u b}+\theta^{l b}}{2 \theta^{h}} .
$$



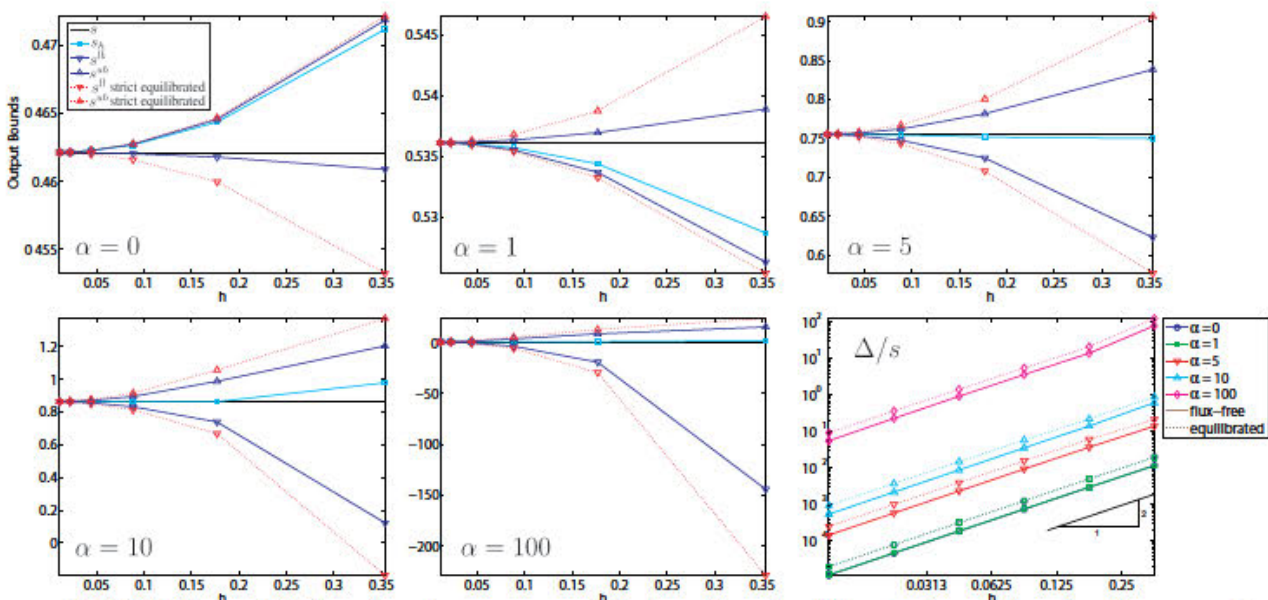

FIG. 7.2. Example 2: bounds and convergence of the bound gap for different values of the advection parameter $\alpha=0,1,5,10$ and 100 for $\sigma=1$.

Again in this example, the flux-free strategy yields much better effectivities that the classical equilibrated fluxes estimates.

\begin{tabular}{cc||cc|cc||cc|c} 
& $n_{\mathrm{el}}$ & $s$ & $s_{h}$ & $s^{l b}$ & $s^{u b}$ & $\theta^{l b}$ & $\theta^{u b}$ & $\eta$ \\
\hline$\alpha=0$ & 312 & 0.290544 & 0.291503 & 0.290315 & 0.291667 & $0.08 \%$ & $0.39 \%$ & $70 \%$ \\
& 734 & & 0.290899 & 0.290446 & 0.290969 & $0.03 \%$ & $0.15 \%$ & $74 \%$ \\
\hline$\alpha=1$ & 312 & 0.328182 & 0.329054 & 0.328050 & 0.329127 & $0.04 \%$ & $0.29 \%$ & $62 \%$ \\
& 473 & & 0.328640 & 0.328081 & 0.328705 & $0.03 \%$ & $0.16 \%$ & $68 \%$ \\
\hline$\alpha=5$ & 312 & 0.478932 & 0.479329 & 0.478407 & 0.479826 & $0.11 \%$ & $0.19 \%$ & $179 \%$ \\
& 402 & & 0.479203 & 0.478642 & 0.479473 & $0.06 \%$ & $0.11 \%$ & $154 \%$ \\
\hline$\alpha=10$ & 312 & 0.618227 & 0.618311 & 0.613727 & 0.622804 & $0.73 \%$ & $0.74 \%$ & $5383 \%$ \\
& 1076 & & 0.618258 & 0.617690 & 0.618793 & $0.09 \%$ & $0.09 \%$ & $1768 \%$ \\
\hline
\end{tabular}

TABLE 7.3

Example 2: Adaptive mesh refinement results for a final tolerance $\Delta_{\text {tol }}=0.001$ s and various values of $\alpha$ and $\sigma=10$ using strict flux-free error estimators.

7.3. Example 3: rotating transport. Again, the steady problem analyzed in [30] is considered. The computational domain is $\Omega=[0,1] \times[0,1]$ and the parameters entering in (2.1) are $\nu=1, \sigma=10$ and $\alpha=500\left(y-\frac{1}{2}, \frac{1}{2}-x\right)$. The boundary conditions are Dirichlet homogeneous on the whole boundary $\partial \Omega$. A localized source term is $f=1000$ in the square $[0.7,0.8] \times[0.7,0.8]$ and $f=0$ elsewhere, see figure 7.3. The output of interest is a local average in the square region $[0.2,0.3] \times[0.2,0.3]$, that is $f^{\mathcal{O}}=1$ in $[0.2,0.3] \times[0.2,0.3]$ and $f^{\mathcal{O}}=0$ elsewhere.

The parameter describing the space discretization is $p=1$ and also in this example the dual estimates are third order polynomials $q=3$. A series of adapted meshes is produced by subdividing at each remeshing step $10 \%$ of the elements, those with the larger contributions to the bound gap, until $\Delta<0.00005$. The adaptive procedure is guided by the indicators (local half bound gap) provided by the strategy proposed in this work. However, in each step, the bounds provided by the strict equilibrated strategy are also computed to compare the results. The initial mesh of 322 elements certifies a wide interval for the 


\begin{tabular}{cc||cc|cc||cc|c} 
& $n_{\mathrm{el}}$ & $s$ & $s_{h}$ & $s^{l b}$ & $s^{u b}$ & $\theta^{l b}$ & $\theta^{u b}$ & $\eta$ \\
\hline$\alpha=0$ & 312 & 0.290544 & 0.291503 & 0.289512 & 0.291779 & $0.36 \%$ & $0.43 \%$ & $118 \%$ \\
& 1114 & & 0.290753 & 0.290298 & 0.290823 & $0.08 \%$ & $0.10 \%$ & $125 \%$ \\
\hline$\alpha=1$ & 312 & 0.328182 & 0.329054 & 0.327359 & 0.329188 & $0.25 \%$ & $0.31 \%$ & $105 \%$ \\
& 853 & & 0.328418 & 0.327907 & 0.328493 & $0.08 \%$ & $0.09 \%$ & $124 \%$ \\
\hline$\alpha=5$ & 312 & 0.478932 & 0.479329 & 0.477642 & 0.480297 & $0.27 \%$ & $0.28 \%$ & $335 \%$ \\
& 1036 & & 0.479038 & 0.478474 & 0.479415 & $0.10 \%$ & $0.10 \%$ & $445 \%$ \\
\hline$\alpha=10$ & 312 & 0.618227 & 0.618311 & 0.609617 & 0.626863 & $1.39 \%$ & $1.40 \%$ & $10227 \%$ \\
& 10619 & & 0.618237 & 0.617644 & 0.618812 & $0.09 \%$ & $0.09 \%$ & $5976 \%$ \\
\hline
\end{tabular}

TABLE 7.4

Example 2: Adaptive mesh refinement results for a final tolerance $\Delta_{\text {tol }}=0.001 \mathrm{~s}$ and various values of $\alpha$ and $\sigma=10$ using strict equilibrated error estimators.

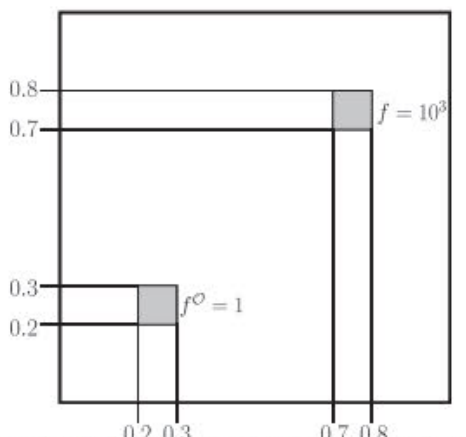

FIG. 7.3. Example 3: Rotating transport forcing and output regions.

quantity of interest $s=0.003526 \pm 0.001337=0.003526 \pm 37.92 \%$, while after remeshing the bounds associated with the final mesh of 3121 elements set a much narrower interval $s=0.003707 \pm 0.000044=0.003707 \pm 1.19 \%$, see table 7.5 and figure 7.4. In each adaptive step, the element size $h$ is computed as $h=\sqrt{1 / n_{\mathrm{el}}}$, and the bounds are compared with the output obtained using a fine mesh (overkill) of 8309 elements $s^{\text {ovk }}=0.00372575$. As in the previous example, the effectivities provided by the flux-free approach are much better than the effectivities provided by the equilibrated method.

\begin{tabular}{c|c||ccc||ccc}
\multicolumn{9}{l||}{} & \multicolumn{3}{c}{ strict equilibrated } \\
\hline$n_{\mathrm{el}}$ & $s_{h}$ & $s^{l b}$ & $s^{u b}$ & $\Delta$ & $s^{l b}$ & $s^{u b}$ & $\Delta$ \\
\hline 322 & 0.003662 & 0.002189 & 0.004864 & 0.00134 & -0.003421 & 0.010602 & 0.00701 \\
666 & 0.003687 & 0.003320 & 0.004018 & 0.00035 & -0.001356 & 0.008683 & 0.00502 \\
1601 & 0.003700 & 0.003599 & 0.003810 & 0.00011 & 0.000751 & 0.006666 & 0.00296 \\
3121 & 0.003707 & 0.003668 & 0.003756 & 0.00004 & 0.001815 & 0.005608 & 0.00190 \\
\hline
\end{tabular}

TABLE 7.5

Example 3: bounds for the initial, intermediate and final meshes of the adaptive h-refinement procedure.

The primal and adjoint solutions are displayed in figure 7.5 for the final mesh, shown in figure 7.6. Also, figure 7.6 shows the local elementary contributions $\Delta_{k}$ to the global bound gap for the initial mesh and for an intermediate mesh of the adaptive procedure. The larger 

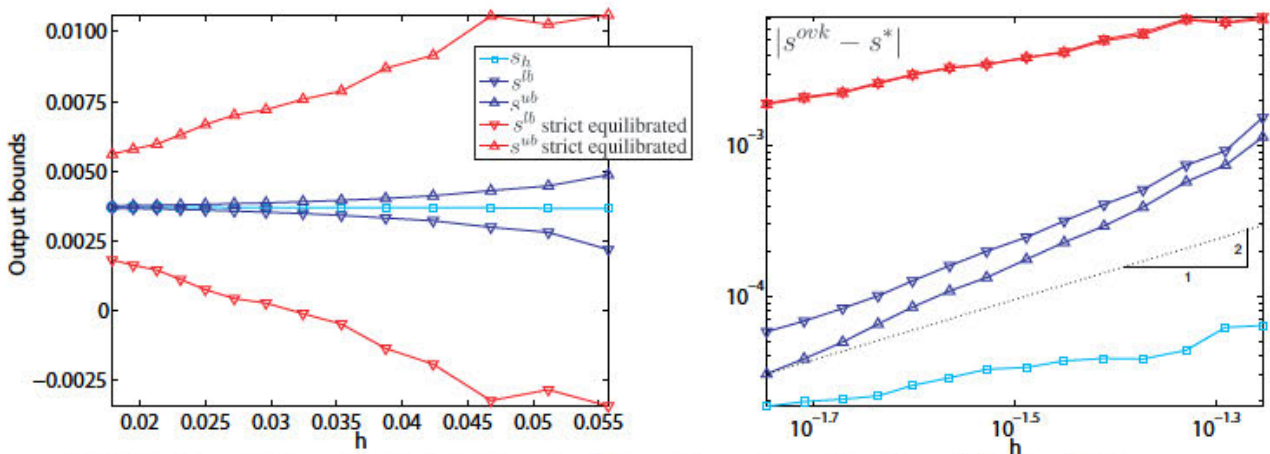

FIG. 7.4. Example 3: series of adapted h-refined linear triangular meshes. Bounds (left) and their convergence (right).

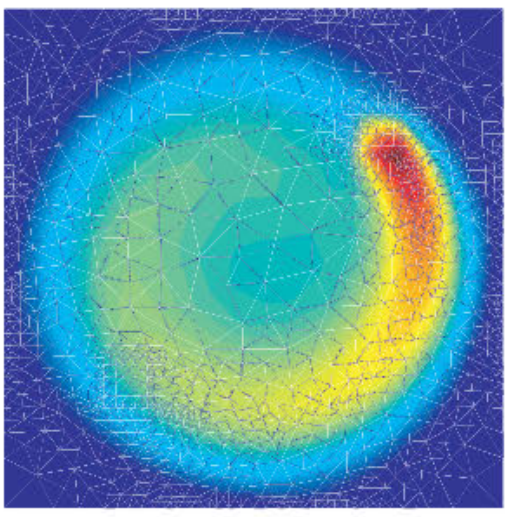

$\begin{array}{llllllll}0.1 & 0.2 & 0.3 & 0.4 & 0.5 & 0.6 & 0.7 & 0.8\end{array}$
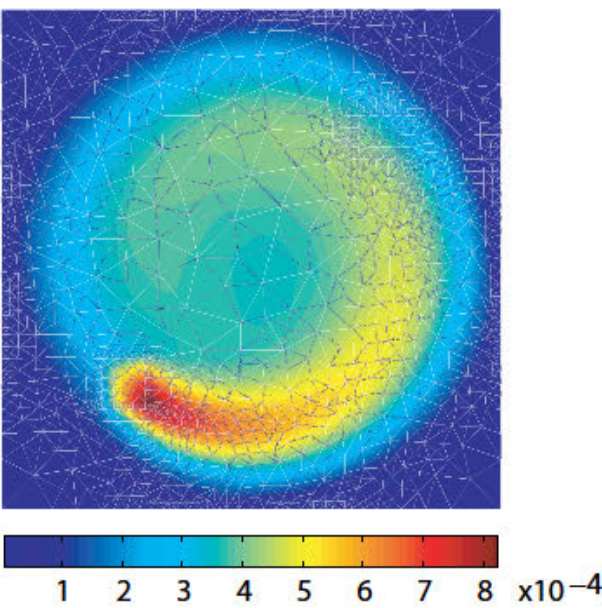

FIG. 7.5. Example 3: Primal and adjoint solutions for the final mesh.

values of the local contributions are precisely in the zones where either the primal or the adjoint solutions have larger gradients.
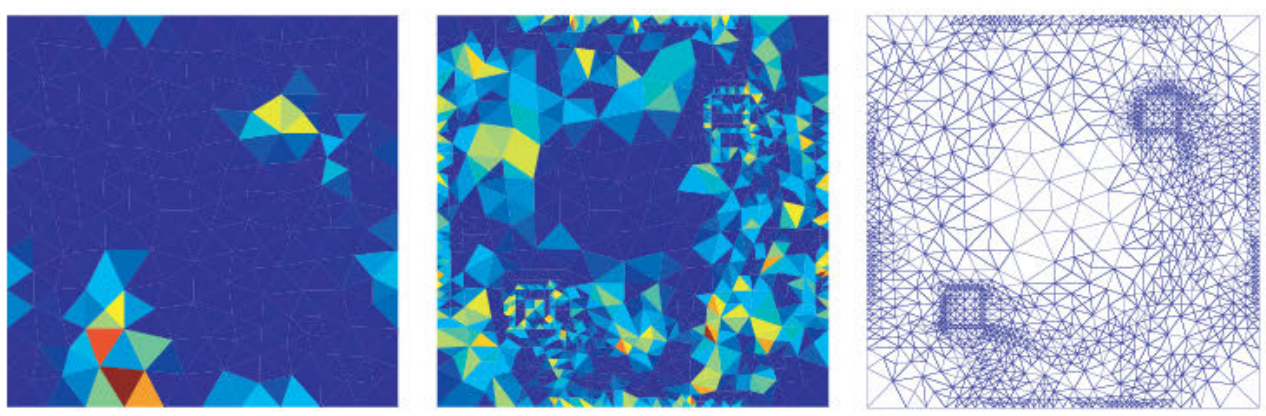

$\begin{array}{llllllllll}0.5 & 1 & 1.5 & 2 & 2.5 & 3 & 3.5 & 4 & 4.5 & 5 \times 10^{-5}\end{array}$

\begin{tabular}{lllll}
1 & 1 & 1 & 1 & 1 \\
\hline & 1 & 2 & 2.5 & $3 \times 10^{-7}$
\end{tabular}

FIG. 7.6. Example 3: Elementary contributions to the half bound gap in initial mesh (left) and intermediate mesh with $n_{\mathrm{el}}=1601$ (center). Final adapted mesh (right). 
7.4. Example 4: solution with parabolic and exponential boundary layers. Here, again an advection-diffusion problem is considered over the domain $\Omega=[0,1] \times[0,1]$. The parameters entering in equation (2.1) are $\nu=10^{-2}, \sigma=0$ and $\alpha=(1,0)$. The boundary conditions are all Dirichlet homogeneous and the source term is $f=1$. The exact solution of the problem is not known analytically. However, the solution possesses a regular boundary layer at the outflow boundary $x=1$ and two parabolic boundary layers at $y=0$ and $y=1$.

The quantity of interest is an overall average of the solution, that is

$$
\ell^{\mathcal{O}}(u)=\int_{\Omega} u(x, y) \mathrm{d} \Omega,
$$

which corresponds to $f^{\mathcal{O}}=1$. For this particular case, $\psi(1-x, y)=u(x, y)$, since the only difference between the primal and adjoint problem is the advection velocity $\alpha$ - the advection velocity associated with the adjoint problem is $(-1,0)$ instead of $(1,0)$. Therefore, the adjoint problem presents the same parabolic boundary layers at $y=0$ and $y=1$, whereas the regular boundary layer is located at $x=0$.

In this example, the adaptive procedure is stopped when a final tolerance $\Delta_{t o l}=0.001$ is reached. At each level of refinement, the elements for which $\Delta_{k} \geq \Delta / n_{\mathrm{el}}$ are refined, where $\Delta$ and $n_{\mathrm{el}}$ are the half bound gap and number of elements at that level. The primal and adjoint solutions obtained for the final mesh are displayed in figure 7.7.
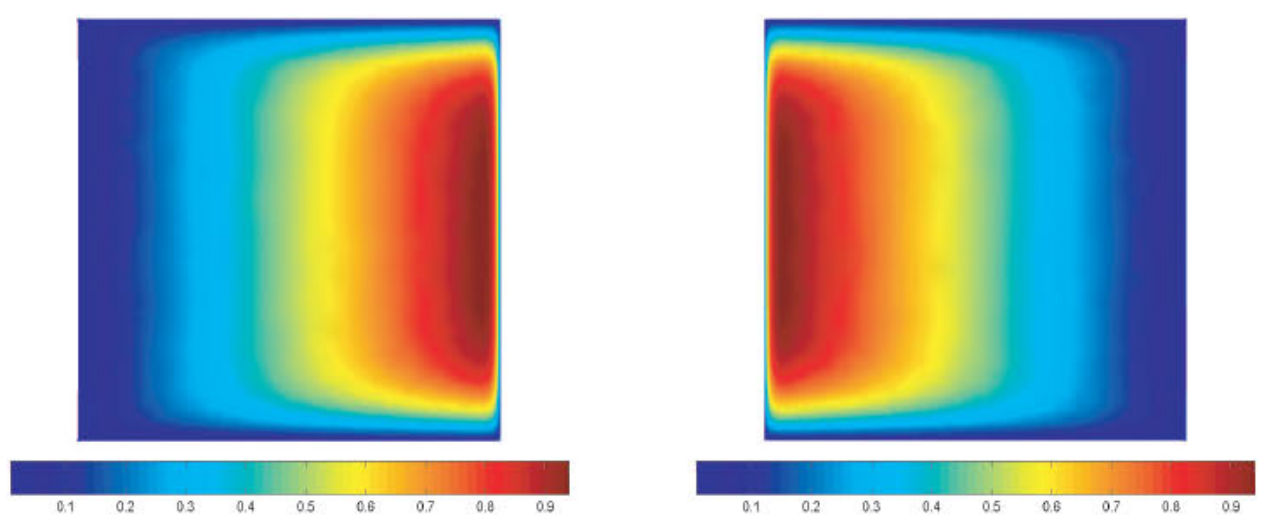

FIG. 7.7. Example 4: Primal (left) and adjoint (right) solutions for the final mesh.

In figure 7.8 the resulting bounds and their convergence are displayed. The initial mesh of 322 elements certifies a wide interval for the quantity of interest $s=0.42728 \pm 0.86897=$ $0.42728 \pm 203.00 \%$, while after remeshing the bounds associated with the final mesh of 31708 elements set a much narrower interval $s=0.43051 \pm 0.00076=0.43051 \pm 0.17 \%$. For the final mesh, also the bounds provided by the equilibrated approach are computed. The guaranteed range for the quantity of interest using this strategy are $s=0.43062 \pm 0.01861=$ $0.43062 \pm 4.32 \%$. Note that although in $2 \mathrm{D}$ the flux-free strategy is computationally more expensive, the bounds that it provides for a given mesh are far more sharp.

Figure 7.9 shows the intermediate meshes verifying that $\Delta<0.2, \Delta<0.05, \Delta<0.005$ and $\Delta<0.001$ respectively, along with their associated primal solutions. It is worth noting that the bounds are guaranteed for all the levels of refinement, even for the meshes where the finite element approximation presents large oscillations. In the initial meshes, the adaptive procedure refines the areas where the primal and adjoint problem present exponential layers to smooth the oscillations of the solutions. However, in order to get tight bounds for the output 

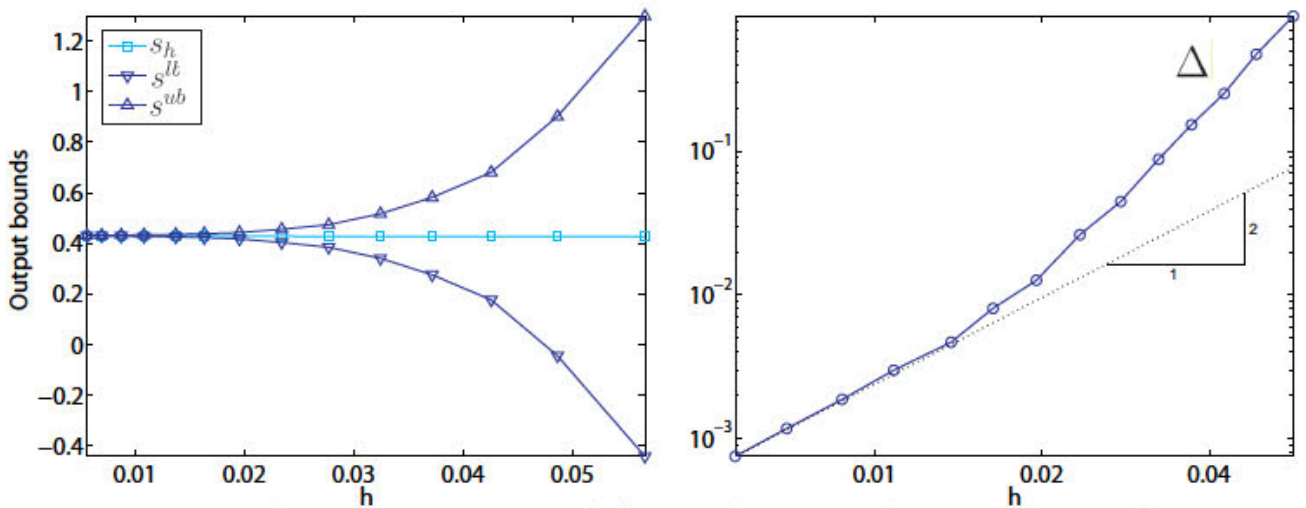

FIG. 7.8. Example 4: series of an adaptively h-refined linear triangular meshes. Bounds (left) and their convergence (right).

also the adaptive procedure has to refine the areas where the solutions present the parabolic layers, as can be seen in the final mesh.
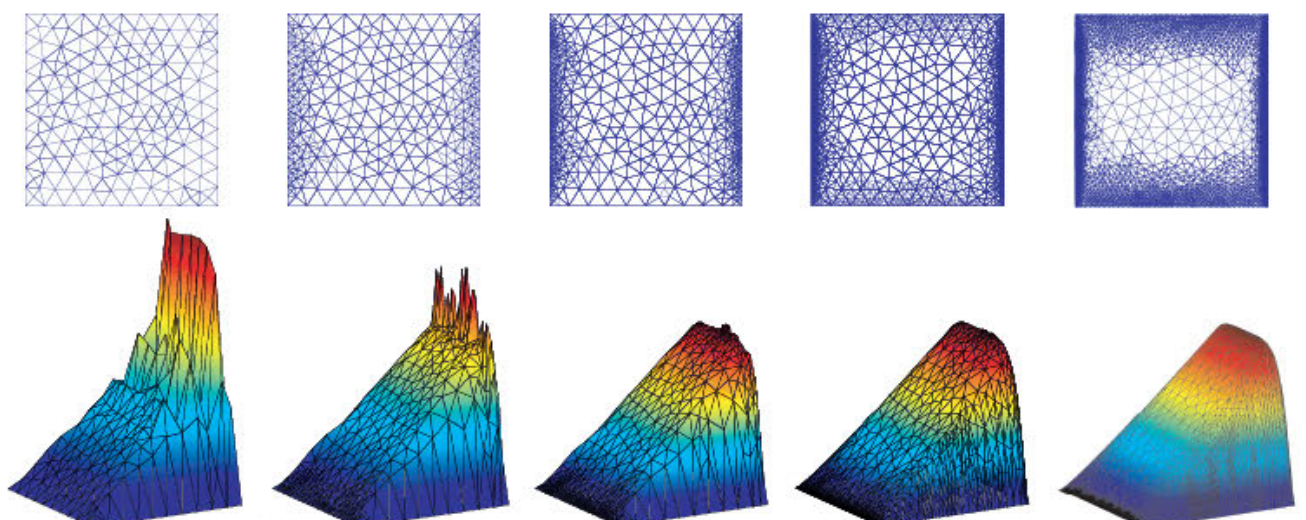
with $\Delta<0.2, \Delta<0.05, \Delta<0.005$ and $\Delta<0.001$ from left to right.

7.5. Example 5: solution with inner layers. Consider the domain $\Omega=[0,1] \times[0,1]$ and the problem given in equation (2.1) with $\sigma=0, \alpha=(y,-x)$, zero source term $f=0$ and for two different values of the diffusion parameter $\nu=10^{-2}$ and $\nu=10^{-3}$. The boundary conditions are Neumann homogeneous on the bottom side $y=0$, and Dirichlet on the remainder sides, homogeneous on the right and top sides $u(1, y)=0$ and $u(x, 1)=0$, and on the left boundary

$$
u(0, y)= \begin{cases}0 & \text { for } \quad|y-0.5|>0.075 \\ 1 & \text { for } \quad|y-0.5|<0.05 \\ 40(y-0.425) & \text { for } \quad 0.425 \leq y \leq 0.45 \\ 40(0.575-y) & \text { for } \quad 0.455 \leq y \leq 0.575\end{cases}
$$

The quantity of interest is again an overall average of the solution, that is

$$
\ell^{\mathcal{O}}(u)=\int_{\Omega} u(x, y) \mathrm{d} \Omega
$$

which corresponds to $f^{\mathcal{O}}=1$. 
The primal and adjoint solutions are displayed in figure 7.10 both for $\nu=10^{-2}$ and $\nu=10^{-3}$. While the primal solution possess a strong inner layer, the adjoint problem has boundary layers at $x=0$ and $y=1$.
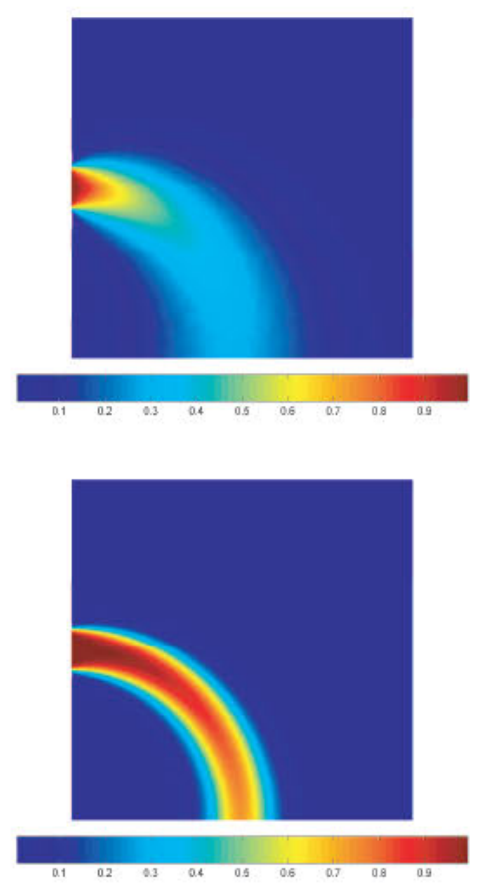
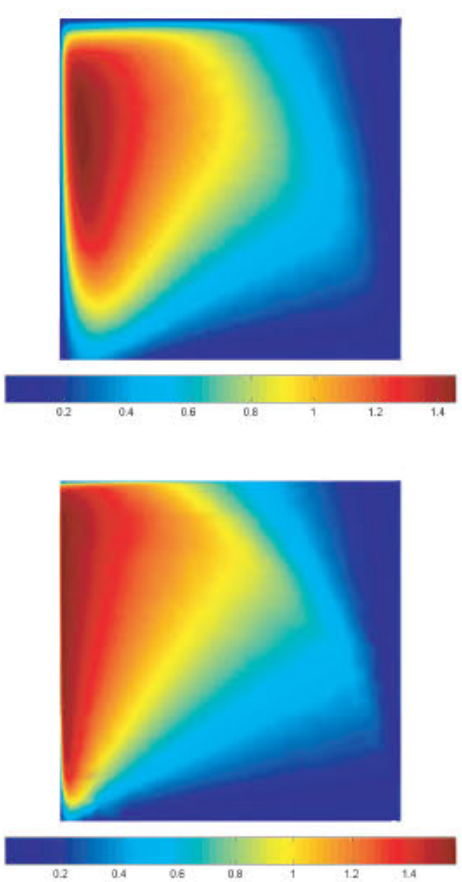

FIG. 7.10. Example 5: Primal (left) and adjoint (right) solutions for the final mesh for $\nu=10^{-2}$ (top) and $\nu=10^{-3}$ (bottom).

The results of the adaptive procedure both for $\nu=10^{-2}$ and $\nu=10^{-3}$ are shown in figure 7.11, where at each level of refinement, the elements for which $\Delta_{k} \geq \Delta / n_{\mathrm{el}}$ are refined, where $\Delta$ and $n_{\mathrm{el}}$ are the half bound gap and number of elements at that level. The initial mesh in both cases contains 672 elements. For the value of the diffusion parameter $\nu=10^{-2}$, the initial mesh certifies that $s=0.09765 \pm 0.02910=0.09765 \pm 29.80 \%$ whereas after remeshing the bounds for a final mesh of 36285 elements set a much narrower interval $s=0.097694 \pm 0.000108=0.097694 \pm 0.11 \%$. For the final mesh also the bounds resulting using the equilibration strategy are computed which yield $s=0.097689 \pm 0.003242=$ $0.097689 \pm 3.32 \%$. Therefore, although the flux-free strategy is more expensive, in order to achieve $\Delta \approx 0.0001$ in the equilibrated strategy, a much more fine mesh would be required, which is far more costly. The results for the smaller diffusion parameter $\nu=10^{-3}$, certify wider intervals for the quantity of interest, as expected since the problem is more convectivedominated and the primal and adjoint solutions require finer meshes to avoid the appearance of oscillatory phenomena. For the initial mesh $s=0.17898 \pm 0.26463=0.17898 \pm 147.86 \%$, while for the final mesh of 37807 elements $s=0.098175 \pm 0.000381=0.098175 \pm 0.38 \%$. Again, the equilibrated strategy provides wider intervals, indeed, $s=0.098182 \pm 0.007960=$ $0.098182 \pm 8.11 \%$ for the final mesh.

Figures 7.12 and 7.13 show some intermediate levels of the adaptive procedure for $\nu=$ $10^{-2}$ and $\nu=10^{-3}$ respectively. In the case $\nu=10^{-2}$, the levels with $\Delta<0.01, \Delta<0.001$, $\Delta<0.0005$ along with the initial and final levels are shown. In the case $\nu=10^{-3}$, the initial and final level are shown along with the levels $\Delta<0.1, \Delta<0.01$ and $\Delta<0.001$. As 

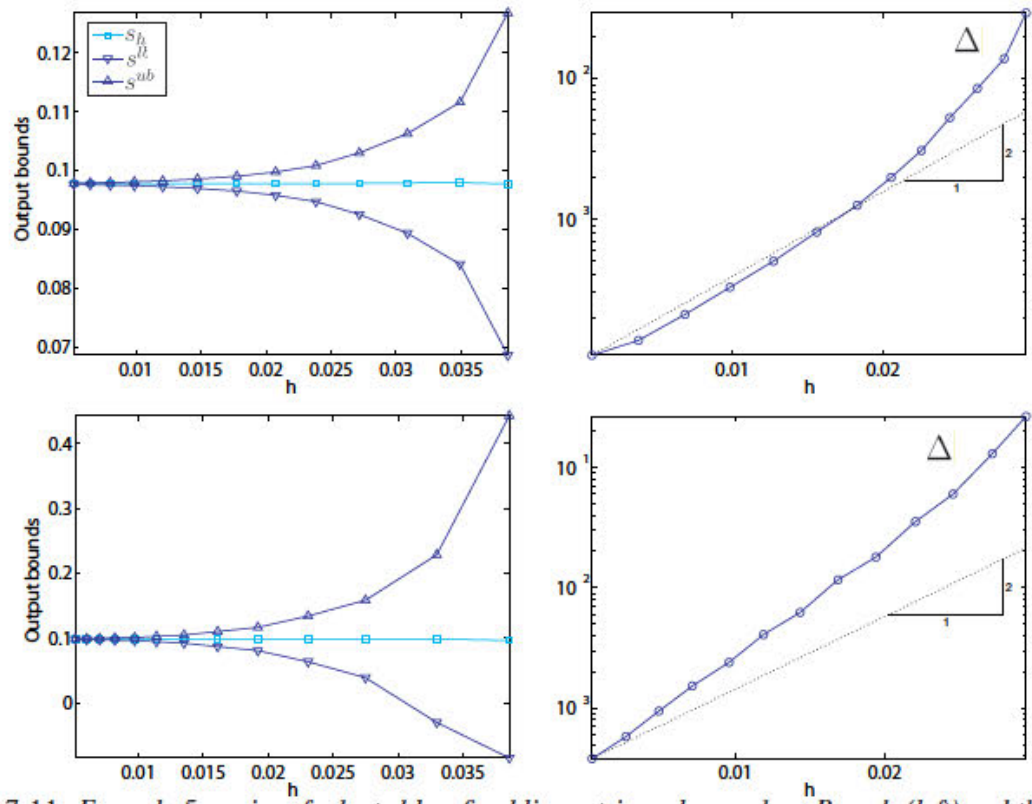

FIG. 7.11. Example 5: series of adapted h-refined linear triangular meshes. Bounds (left) and their convergence (right) for $\nu=10^{-2}$ (top) and $\nu=10^{-3}$ (bottom).

the diffusion coefficient $\nu$ is decreased, and therefore, the problem becomes more advection dominated, the inner and boundary layers are more stiff and therefore the adaptive strategy requires more steps in order to smooth out the oscillations appearing in the primal and adjoint solutions. However, even for non converged solutions, the resulting bounds are guaranteed.

8. Concluding remarks. This paper introduces a new technique to compute strict upper and lower bounds for functional outputs. The bounds are guaranteed regardless of the size of the underlying coarse discretization and do not involve uncertain ingredients (in some cases, the bounds may depend on continuity or interpolation constants which are non-computable and have to be approximated, or they may depend on the evaluation of integrals involving analytic functions which have to be numerically integrated ... ).

The proposed strategy is an extension of the flux-free technique presented in [22]. In [22], the resulting estimates yield upper bounds of a reference solution associated with a finer reference mesh. This bounds are only strict provided that the error associated to the reference solution is negligible in front of the error associated with the coarse solution. In the present work, this assumption is removed, and the bounds are strict without any further assumption.

The observed accuracy of the flux-free approach is much better than in the hybrid-flux methods. Also, the new flux-free technique yield much sharper bounds than other previously proposed flux-free techniques.

It is worth noting that although the strategy may be applied to advection-dominated problems, the effectivities of the bounds deteriorate as the advection term becomes dominant. This phenomenon is due to the expression of the bounds of the output given in theorem 3.1, and is also observed in all the previous works which compute strict or asymptotic bounds for quantities of interest for the advection-reaction-diffusion equation. Sharper bounds which partially overcome this behavior may be obtained using the approach proposed in [20] together with the strategy presented in [8]. Moreover, the generalization of this technique to stabilized methods [9] is also expected to alleviate this problem.

Finally, the distribution of the local contributions to the error are well suited to guide 

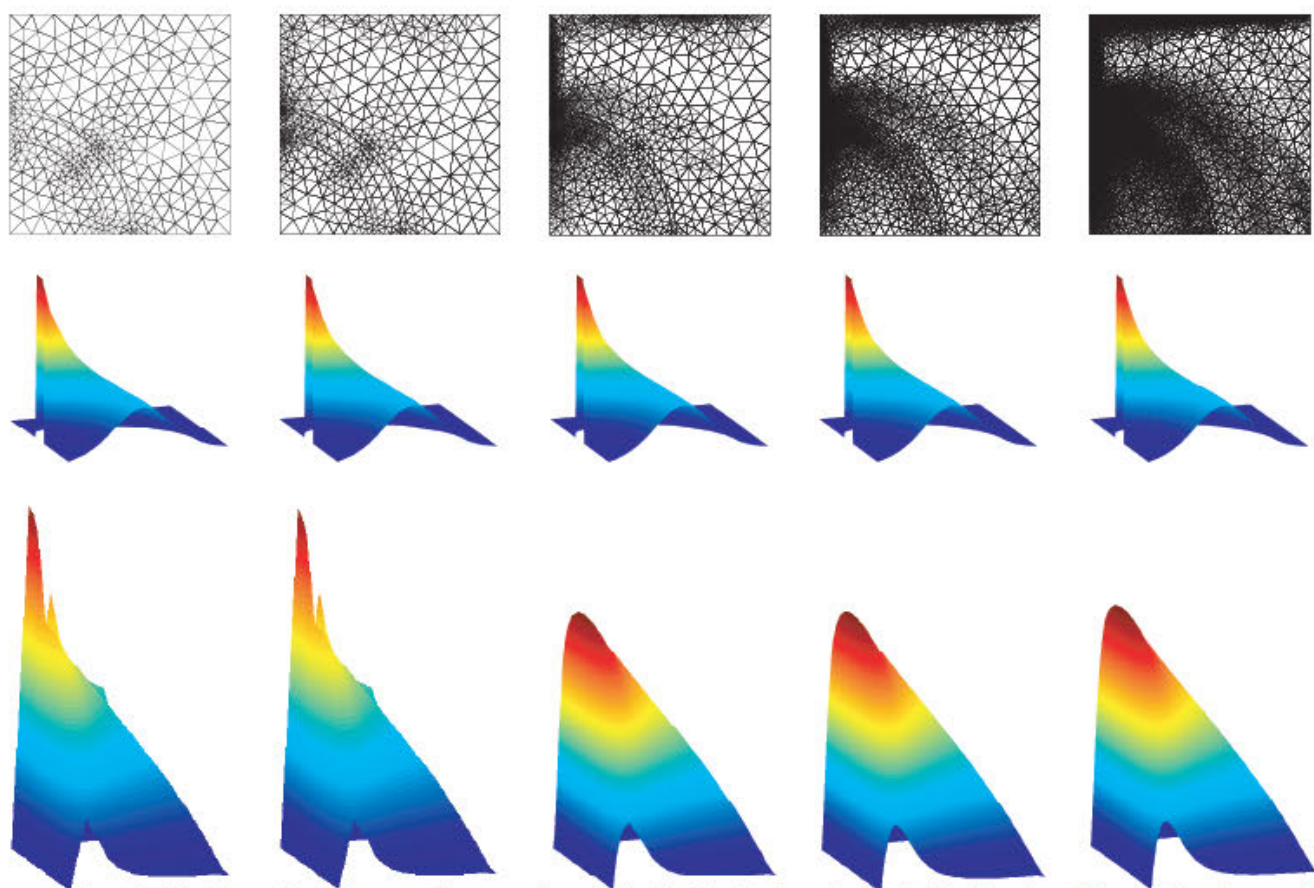

FIG. 7.12. Example 5: intermediate meshes and primal solutions for the initial level and for the levels associated with $\Delta<0.01, \Delta<0.001, \Delta<0.0005$ and for the final mesh $\Delta \approx 0.0001$.
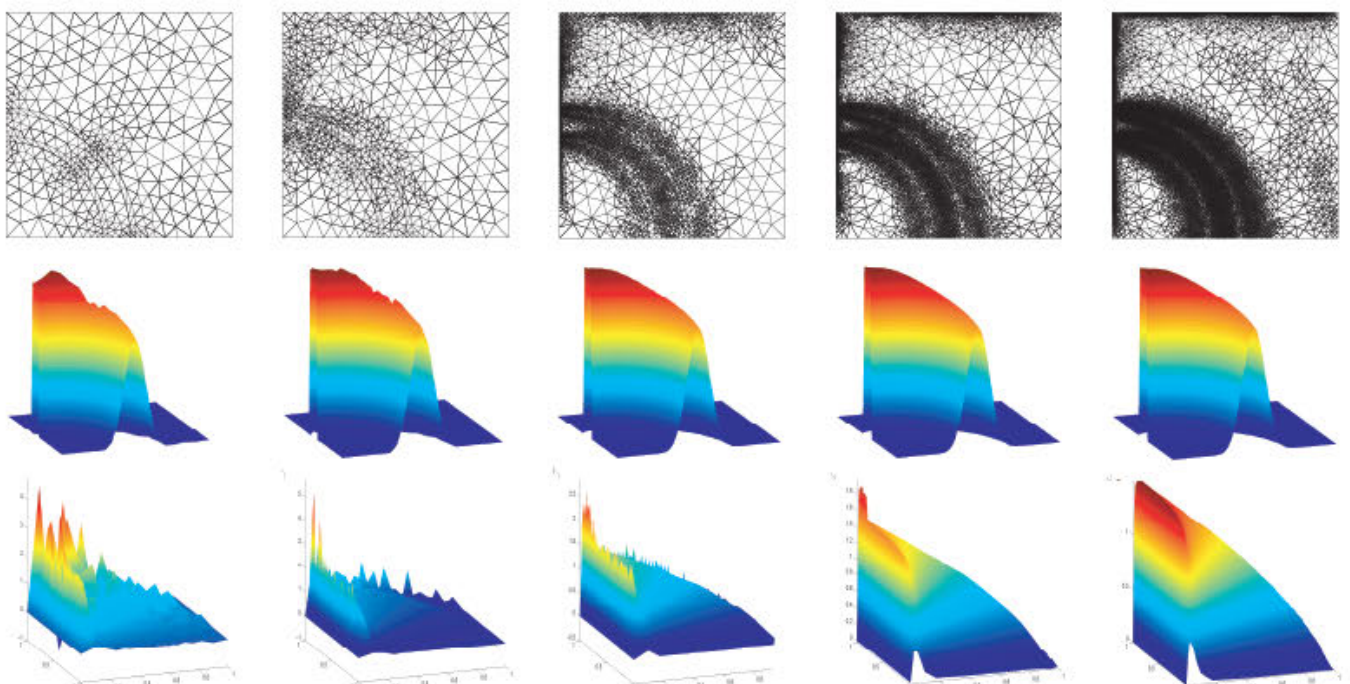

FIG. 7.13. Example 5: intermediate meshes and primal solutions for the initial level and for the levels associated with $\Delta<0.1, \Delta<0.01$ and $\Delta<0.001$ and for the final mesh $\Delta<0.0005$.

goal-oriented adaptive procedures.

\section{REFERENCES}

[1] M. AINSWORTH AND I. BABUŠKA, Reliable and robust a posteriori error estimating for singularly perturbed reaction-diffusion problems, SIAM J. Numer. Anal., 36 (1999), pp. 331-353 (electronic).

[2] M. Ainsworth AND J. T. Oden, A posteriori error estimation in finite element analysis, John Wiley \& Sons, Chichester, 2000. 
[3] I. BABuŠKa And T. Strouboulis, The finite element method and its reliability, Numerical Mathematics and Scientific Computation, The Clarendon Press Oxford University Press, New York, 2001.

[4] R. BECKER AND R. RANNACHER, An optimal control approach to a posteriori error estimation in finite element methods, Acta Numer., 10 (2001), pp. 1-102.

[5] D. BRAess AND J. Schoberl, Equilibrated residual error estimator for edge elements, Math. Comp., 77 (2008), pp. 651-672.

[6] C. CARstensen And S. A. Funken, Fully reliable localized error control in the FEM, SIAM J. Sci. Comput., 21 (1999/00), pp. 1465-1484 (electronic).

[7] P. DestUYNDER AND B. MÉTIVET, Explicit error bounds in a conforming finite element method, Math. Comp., 68 (1999), pp. 1379-1396.

[8] P. DÍEZ, N. PARÉS, AND A. HUERTA, Recovering lower bounds of the error by postprocessing implicit residual a posteriori error estimates, Internat. J. Numer. Methods Engrg., 56 (2003), pp. 1465-1488.

[9] J. Donea And A. Huerta, Finite Element Methods for Flow Problems, John Wiley \& Sons, Chichester, 2003.

[10] M. B. GILES AND E. SÜLI, Adjoint methods for PDEs: a posteriori error analysis and postprocessing by duality, Acta Numer., 11 (2002), pp. 145-236.

[11] S. KoROTOV, Two-sided a posteriori error estimates for linear elliptic problems with mixed boundary conditions, Appl. Math., 52 (2007), pp. 235-249.

[12] M. KŘ́̌̌ž Conforming equilibrium finite element methods for some elliptic plane problems, RAIRO Anal. Numér., 17 (1983), pp. 35-65.

[13] P. LAdEVÈze AND D. LEGUILLON, Error estimate procedure in the finite element method and applications, SIAM J. Numer. Anal., 20 (1983), pp. 485-509.

[14] R. LuCE AND B. I. Wohlmuth, A local a posteriori error estimator based on equilibrated fluxes, SIAM J. Numer. Anal., 42 (2004), pp. 1394-1414 (electronic).

[15] L. Machiels, Y. MAdAY, AND A. T. PATERA, A “flux-free" nodal Neumann subproblem approach to output bounds for partial differential equations, C. R. Acad. Sci. Paris Sér. I Math., 330 (2000), pp. 249-254.

[16] P. MoRin, R.H. NocheTto, AND K.G. SiEBERT, Local problems on stars: a posteriori error estimators, convergence, and performance, Math. Comp., 72 (2003), pp. 1067-1097.

[17] J. T. ODEN AND S. PRUDHOMme, Goal-oriented error estimation and adaptivity for the finite element method, Comput. Math. Appl., 41 (2001), pp. 735-756.

[18] M. PARASCHIVOIU AND A. T. PATERA, A hierarchical duality approach to bounds for the outputs of partial differential equations, Comput. Methods Appl. Mech. Engrg., 158 (1998), pp. 389-407.

[19] M. PARAschivoiu, J. Peraire, AND A. T. PATERA, A posteriori finite element bounds for linear-functional outputs of elliptic partial differential equations, Comput. Methods Appl. Mech. Engrg., 150 (1997), pp. 289-312. Symposium on Advances in Computational Mechanics, Vol. 2 (Austin, TX, 1997).

[20] N. PARÉS, Error assessment for functional outputs of PDE's: bounds and goal-oriented adaptivity, PhD thesis, Technical University of Catalonia (UPC), January 2005.

[21] N. Parés, J. Bonet, A. Huerta, And J. Peraire, The computation of bounds for linear-functional outputs of weak solutions to the two-dimensional elasticity equations, Comput. Methods Appl. Mech. Engrg., 195 (2006), pp. 406-429.

[22] N. PARÉS, P. DÍEZ, AND A. HUERTA, Subdomain-based flux-free a posteriori error estimators, Comput. Methods Appl. Mech. Engrg., 195 (2006), pp. 297-323.

[23] - Bounds of functional outputs for parabolic problems. Part I: Exact bounds of the discontinuous galerkin time discretization, Comput. Methods Appl. Mech. Engrg., 197 (2008), pp. 1641-1660.

[24] - Bounds of functional outputs for parabolic problems. Part II: Bounds of the exact solution, Comput. Methods Appl. Mech. Engrg., 197 (2008), pp. 1661-1679.

[25] N. PARÉs, H. SANTOS, AND DÍEZ P., Guaranteed energy error bounds for the Poisson equation using a flux-free approach: solving the local problems in subdomains. Accepted for publication in Internat. J. Numer. Methods Engrg..

[26] S. PRUDHOMME AND J. T. ODEN, Computable error estimators and adaptive techniques for fluid flow problems, in Error estimation and adaptive discretization methods in computational fluid dynamics, vol. 25 of Lect. Notes Comput. Sci. Eng., Springer, Berlin, 2003, pp. 207-268.

[27] H. J. REINHARDT, A posteriori error analysis and adaptive finite element methods for singularly perturbed convection-diffusion equations, Math. Methods Appl. Sci., 4 (1982), pp. 529-548.

[28] S. REPIN AND S. SAUTER, Functional a posteriori estimates for the reaction-diffusion problem, C. R. Math. Acad. Sci. Paris, 343 (2006), pp. 349-354.

[29] A. M. Sauer-Budge, J. Bonet, A. Huerta, And J. Peraire, Computing bounds for linear functionals of exact weak solutions to Poisson's equation, SIAM J. Numer. Anal., 42 (2004), pp. 1610-1630 (electronic).

[30] A. M. SAUER-Budge AND J. Peraire, Computing bounds for linear functionals of exact weak solutions to the advection-diffusion-reaction equation, SIAM J. Sci. Comput., 26 (2004), pp. 636-652 (electronic).

[31] T. Strouboulis AND J. T. Oden, A posteriori error estimation of finite element approximations in fluid 
mechanics, Comput. Methods Appl. Mech. Engrg., 78 (1990), pp. 201-242.

[32] T. Strouboulis, L. Zhang, D. WANG, AND I. BABUŠKA, A posteriori error estimation for generalized finite element methods, Comput. Methods Appl. Mech. Engrg., 195 (2006), pp. 852-879.

[33] W. G. SZYMCZAK AND I. BABUŠKA, Adaptivity and error estimation for the finite element method applied to convection diffusion problems, SIAM J. Numer. Anal., 21 (1984), pp. 910-954.

[34] T. VeJCHODSKÝ, Guaranteed and locally computable a posteriori error estimate, IMA J. Numer. Anal., 26 (2006), pp. 525-540.

[35] R. VERFÜRTH, Robust a posteriori error estimators for a singularly perturbed reaction-diffusion equation, Numer. Math., 78 (1998), pp. 479-493.

[36] - Robust a posteriori error estimates for nonstationary convection-diffusion equations, SIAM J. Numer. Anal., 43 (2005), pp. 1783-1802 (electronic).

[37] - Robust a posteriori error estimates for stationary convection-diffusion equations, SIAM J. Numer. Anal., 43 (2005), pp. 1766-1782 (electronic).

[38] M. Vohralík, A posteriori error estimation in the conforming finite element method based on its local conservativity and using local minimization, C. R. Math. Acad. Sci. Paris, 346 (2008), pp. 687-690.

[39] Z. C. XuAn, N. PARÉS, AND J. Peraire, Computing upper and lower bounds for the J-integral in twodimensional linear elasticity, Comput. Methods Appl. Mech. Engrg., 195 (2006), pp. 430-443. 